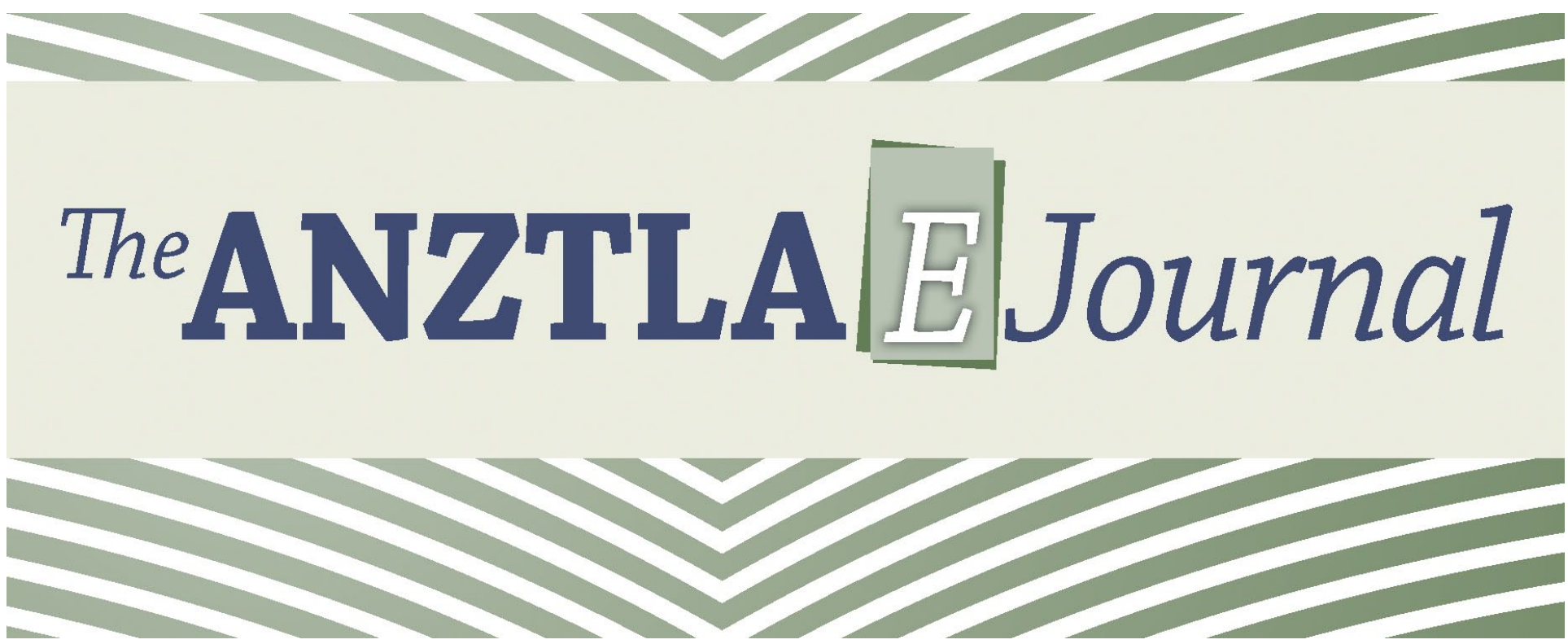

Australian and New Zealand Theological Library Association W No. 14 (2015)

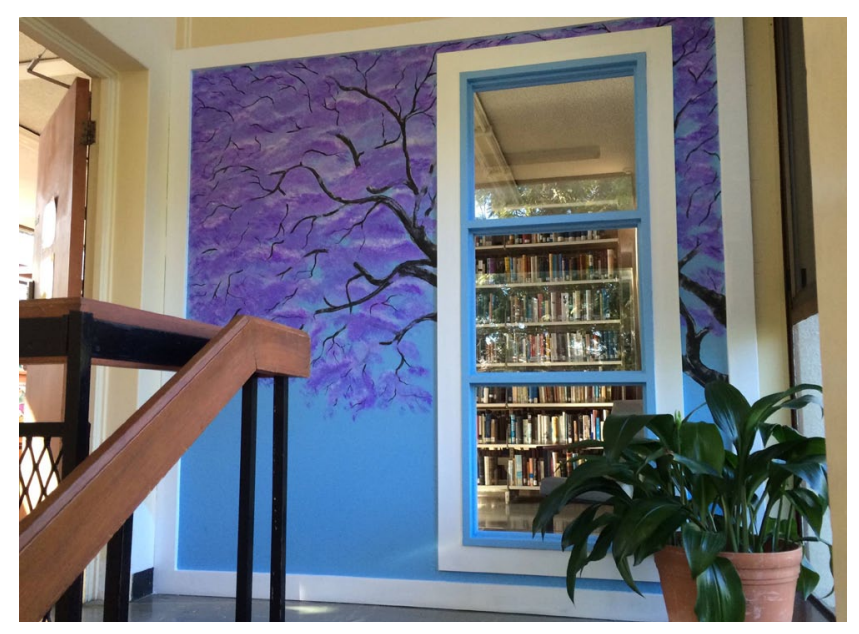

Entrance to the Roscoe Library at St Francis Theological
W elcome to the fourteenth issue of The ANZTLA EJournal - the official serial publication of the Australian and New Zealand Theological Library Association. The ANZTLA EJournal is indexed in the Australasian Religion Index (ARI).

Chapter reports are useful. They can provide us with a sense of satisfaction of a job well done, or give inspirational ideas for practices or improvements that can be incorporated into our own libraries.

ANZTLA statistics track our progress and provide us with data that can be used to advocate for increased services or resources. Use them well!

\section{Eve James}

\section{Editor}

\title{
Contents
}

2 ANZTLA SA Chapter Report 2014

7 ANZTLA Queensland Chapter Report 2014

9 ANZTLA 2014 Statistics

23 Contributors 


\section{ANZTLA South Australia Chapter Report 2014 \\ compiled by Hayley Eyers, ANZTLA SA Chapter Secretary}

\section{Current financial South Australian Members of ANZTLA (2014)}

- Adelaide College of Ministries Library, Adelaide College of Ministries

- Adelaide Theological Library, Adelaide College of Divinity

- Australian House of Prayer for All Nations Library, Australian House of Prayer for All Nations

- Burrow Library, Bible College of South Australia

- Catholic Resource \& Information Service, Catholic Archdiocese of Adelaide

- Löhe Memorial Library, Australian Lutheran College

- Sophia Library, Sophia

- Tabor Adelaide Library, Tabor Adelaide

\section{News from the ANZTLA SA Chapter}

- Barbara Cooper was elected as new Chairperson for ANZTLA SA Chapter.

- Blan MacDonagh, library manager at Löhe Memorial Library, Australian Lutheran College, retired in November 2014.

- ANZTLA SA Chapter has been coming up with new ideas for future meetings, for example, library tours and workshops.

- ANZTLA SA toured the new Adelaide City Library and South Pacific School Aid this year.

- Sophia Library joined the ANZTLA SA Chapter. The Sophia Library is a public library with a specialist collection of fiction and non-fiction books, poetry, periodicals, DVDs, and CDs for loan and for reference. Subject areas include psychology, feminist theology, women's studies, spiritual life, meditation and ritual.There are also books on feminist, social, health and ecological concerns and social justice issues in addition to small history, fiction and poetry sections. The Sophia Library is an important resource in the life of Sophia. People who attend courses value the opportunity to browse and borrow relevant items. Specific books, which are recommended by course facilitators, are also purchased for the Library.

\section{Adelaide's City Library}

Opened on Friday 7 February 2014, the City Library is a multipurpose space. It is a place of possibility, where creativity and innovation are fostered through cutting edge technologies, inspirational spaces and functional universal design. People will meet in this space, they will 
"An overarching design principle for the City Library is the concept of 'one element many forms', guiding the creation of flexible and multipurpose spaces, and the selection of joinery and furniture that could be used by our community in multiple ways." hang out, learn, create and debate. They will access Adelaide's history and be part of Adelaide's future.

Adelaide's City Library will have enough room for big ideas and small. It will be central to the cultural calendar of Adelaide and readers, writers, makers and performers will all embrace this flexible space and help make the City Library the place to be.

Recently the City Library won an Excellence in Design award from the Forest Stewardship Council (FSC) - Australia.

The FSC is an international, non-profit organisation founded in 1993 by environmentalists, social interest groups, indigenous peoples' organisations, responsible retailers and forest management companies to develop standards to measure responsible forest management practices.

The City Library team received this prestigious award for the design, selection and procurement of responsibly sourced, FSCcertified timber products for many of its fixtures, finishes and furniture, as well as FSC-certified paper for its office and sanitary supplies. The City Library sustainable design principles reflect what is important in our city and to our community.

An overarching design principle for the City Library is the concept of "one element many forms", guiding the creation of flexible and multipurpose spaces, and the selection of joinery and furniture that could be used by our community in multiple ways.

This award recognises that Adelaide City Council is committed to promoting public spaces that are socially, culturally, economically and environmentally sustainable.

\section{South Pacific School Aid}

South Pacific School Aid is a group of volunteers who collect, sort, pack and ship mainly second hand books, suitable for educational purposes, originally to countries of the South Pacific but, in recent years, to many places all over the world. (The group has existed for more than thirty years.)

Recently all records were broken with a large shipment of 71 pallets containing more than 90,000 books being sent to 24 locations.

Peter Kirk, who leads the committee, is constantly in contact with schools, charity clubs and churches, not only sourcing books but also funds to ship pallets and containers all around the world, while David Butler spends many hours preparing and painting the boxes with the red kangaroo so that overseas people recognise they come from Australia. Books that do not make the grade for shipment overseas are sold here in Australia to help raise funds for each shipment.

It is not surprising that this group was presented by the Governor of South Australia with the Adelaide Chapter Achievement Award of 2012 UNESCO Clubs. This wonderful group deserves such a prestigious award. 
"South Pacific

School Aid is a

group of volunteers

who collect, sort,

pack and ship

mainly second hand

books, suitable

for educational

purposes, originally

to countries of the

South Pacific but,

in recent years, to

many places all over

the world."

\section{Adelaide College of Ministries Library}

The Adelaide College of Ministries Library has had another quiet year of business as usual. We contemplated extending our Online Journals subscription this year to take in the ATLA range, but have deferred that until more funds are available. However we were excited to receive into the collection a large set of Loeb Classical Library items as well as some quality donations. We have also been able to undertake a more detailed stocktake this year and so have a much more confident understanding of the content of the collection and where we need to extend. Developing an acquisitions policy remains on the strategic priority list.

\section{Tamara Wenham}

\section{Adelaide Theological Library}

The Adelaide Theological Library is now operating under a new management body, Adelaide Theological Library Inc. The new Board includes representation from the Adelaide College of Divinity, the Uniting College, the SA Synod of the Uniting Church, and Flinders University Library. In effect, the managing body will continue operating as before under a new name except with no responsibility for the campus itself, as this is now solely owned by the Uniting Church. The resignations from the consortium by the colleges of the Anglican Church and the Catholic Church have led to a significant reduction in staffing levels, acquisitions budgets and opening hours.

Rosemary Hocking

\section{Diocesan Archives, Anglican Diocese of Adelaide}

In October 2014 Adelaide's longstanding Anglican Diocesan archivist, Mrs Robin Radford, entered retirement. Her position has been filled by Dr Sarah Black. The archives spent 2014 in a busy period of consolidation, working on a program of reorganising the collection to embrace the series system as well as processing some large new accessions. We expect an eventful year in 2015, including a move to new premises located together with St Barnabas Theological College. With the impending resurrection of the St Barnabas Theological College library, we are looking forward to exploring possibilities for fruitful collaboration as time goes on.

Sarah Black, Diocesan Archivist

\section{Burrow Library}

First semester saw the welcome addition of three casual volunteers, one of whom has previous experience as a librarian. This has helped greatly with the sorting and cataloguing of donated books in particular. In the middle of the year we upgraded our library management system from ALICE to Liberty. This followed outsourcing of the college server and IT management. There have been the inevitable hiccoughs but generally the result of this upgrade 
"The rest of the year was occupied by, as well as our usual tasks, the digitalisation of the illustrations of the Luther Bible overseen by Trevor Schaefer" has been pleasing and facilitates online access to resources for our students.

Barbara Cooper

\section{Catholic Resource \& Information Service}

This year the emphasis at CRIS has been to respond to the initiatives and changes within the Catholic Education Office while continuing to support Graduate Certificate courses, APRIMS and RECs in schools and developments within Parish Catechesis and Youth Ministry. There has been strong emphasis on the Theology of the Child, ecological conversion, changes to Grad Cert and Catholic Studies courses due to the migration of Grad Cert to UniSA, and more interaction with ACU students and staff due to integration of Catholic Theological College into Australian Catholic University and co-location with Catholic Education SA. Susan attended the National ACRATH Conference in Melbourne in February, and has promoted awareness of human trafficking issues. 2015 will see an upgrade of the CRIS library management system to the cloud based Infiniti, and further modifications to the delivery of courses.

\section{Löhe Memorial Library}

There has been much activity at LML this year especially in the latter part when our usual calm existence was somewhat rippled by Blan MacDonagh, our Library Manager for 10 years, announcing her retirement. She left us in November accompanied by appreciation for the work she had done. Her resignation was followed by a review of the Library conducted by former ATL Librarian, Beth Prior. The results of the report have not been widely distributed but will inform the appointment of a 'Senior Librarian' early in 2015. Lavinia Gent has been acting as Library Manager in an interim capacity.

Also towards the end of the year, the Library was given access to a significant bequest to buy computer hardware and furniture for the setting up of a Computer Lab. We hope this project will be completed early in 2015. The rest of the year was occupied by, as well as our usual tasks, the digitalisation of the illustrations of the Luther Bible overseen by Trevor Schaefer, and the weeding of our video collection which was replaced, where possible, with DVDs and other digital forms by Pam Zweck Silcock. Lavinia as Cataloguer continues to come to terms with the implications of RDA and also oversaw the upgrade of our Symphony Library Management System. 2014 was a significant year for us and we look forward to new challenges (and some old routines) in 2015.

Lavinia Gent, Interim Library Manager

\section{Tabor Adelaide Library}

2014 was a year of significant and exciting achievements for the Tabor Adelaide Library. The implementation of a new library system, Aleph/Primo in October 2014 was the biggest change and 
major milestone that required a great deal of planning, preparation and training. The new catalogue offers an easy electronic resource access to journals and e-books and faceted searching. Students and academic staff were randomly surveyed at the end of the year, and the feedback was overwhelmingly positive. Tabor Adelaide is now a member of a UNILINC library network that has over 22 academic libraries sharing support and resources via interlibrary loans.

Oksana Feklistova

"2014 was a year

of significant

and exciting

achievements for

the Tabor Adelaide

Library. The

implementation

of a new library

system, Aleph/

Primo in October

2014 was the

biggest change

and major

milestone that

required a great

deal of planning,

preparation and

training." 


\section{ANZTLA Queensland Chapter Report 2014}

$\mathrm{L}$

ibraries under construction! Building in progress! - That's the report in brief from all the ANZTLA Q members this year.

\section{Real and virtual!}

Not only is the Queensland Theological College planning a new bricks and mortar library, but for most of our members, virtual walls have expanded with ebooks added to traditional collections. In the words of Annette McGrath, "2014 has been a year of building, building collections and building for the future”. In building our virtual libraries, we've found that the convenience of ebooks far outweighs initial complications with authentication and access.

A new Principal at the Brisbane School of Theology has encouraged the substantial acquisition of current publications, especially in New Testament studies. After describing the fine religious art collection donated to the Roscoe Library, Eve enthused that: "It's been a full-on year but a good one" at St Francis. Donations to the Malyon College Library will be catalogued first before shipping them to a theological college in the Solomon Islands at the beginning of next year. Ian Stoodley is helping Jill with this big job. Meanwhile, ACU have decided to no longer take donations. St Paul's Seminary is being redeveloped to include an archive. The transfer of archival material is partially done and will continue next year.

The Sunshine Coast Theological College (STC) Library subscribed to ATLA for the first time this year and the Brisbane School of Theology (BST) is planning to do so. STC and BST are both in the process of planning new library management systems. The choices are Koha or Liberty.

\section{New \& renewed!}

'It's a great job," says Stephen Morton at Christian Heritage College (CHC), where he's restructured staffing and opening hours; and at Trinity there's a new Learning \& Teaching Librarian, a job shared by Anna Lagos and Lyndelle Gunton. Joining ANZTLA this year was only part of Nica's 'rollercoaster year' as the newly appointed liaison librarian in theology at ACU. Our bright and colourful Sarah Howard left us for a new team at Queensland University of Technology.

\section{Show \& tell!}

In contrast to Kevin Hannah's anecdotes at the 2014 ANZTLA conference, CHC now raves about Steve's innovative displays and Trinity has brash new orange LIBRARY signage. Eventbrite is being successfully used by St Francis and ACU for promotional purposes. Annette's been inspired with new ideas after her attendance at the ATLA Conference in New Orleans. The local chapters of ANZTLA and ANZATS met together twice this 
year to exchange ideas and, at the last meeting, Trinity and ACU librarians presented their stories on how librarians are supporting online learning environments.

We're happy with that: Andree Pursey ( Nazarene Theological College), Angela Gear (Sunshine Coast Theological College), Annette McGrath (Queensland Theological College), Eve James \& Susan Thomas (St Francis Theological College), Helen Johnston (Brisbane School of Theology), Jill Walker (Malyon College), Nica Tsakmakis (Australian Catholic University), Stephen Morton (Christian Heritage College), Alethea Hubley, Anna Lagos, and Lyndelle Gunton (Trinity Theological Library).

"In building our virtual libraries, we've found that the convenience of ebooks far outweighs initial complications with authentication and access." 


\title{
ANZTLA 2014 Statistics
}

\author{
by Kerrie Stevens
}

\section{ANZTLA Statistician}

\footnotetext{
NZTLA Statistics are compiled annually from data provided by ANZTLA member libraries on a voluntary basis.
}

The reporting categories are described as follows:

- A Provides clergy training programmes (ie: The Australian and New Zealand Association of Theological Schools [ANZATS] member schools) and/or schools and training institutions which offer broad theological training (ie: Bible Colleges, Missionary Training Institutions) possibly leading to degree or post-graduate qualifications;

- B Do not have students (ie: church administrative libraries, resource centres, parachurch organisations, etc.); and

- C Institutions offering non-theological courses in addition (ie: teacher training).

Data has been entered as submitted by the libraries. Any questions should be directed to the libraries concerned.

Thank you to all libraries who contribute to the ANZTLA Statistics!

Kerrie Stevens has also supplied two supplementary fles to accompany the statistics. She produced a map using Google Maps to indicate the location of each library who responded to the call for Statistics. The second file features the 2014 ANZTLA Statistics report. 


\section{TABLE 12014 LIBRARY INFORMATION}

\begin{tabular}{|c|c|c|c|}
\hline $\begin{array}{l}0 \\
0 \\
\infty\end{array}$ & INSTITUTION & $\begin{array}{l}\text { STATE/N } \\
z\end{array}$ & $\begin{array}{l}\text { PREDOMINANT DENOMINATION OF } \\
\text { COLLECTION }\end{array}$ \\
\hline A & Adelaide College of Divinity - Adelaide Theological Library & SA & Well-spread over all denominations \\
\hline $\mathrm{C}$ & Alphacrucis College - James Wallace Memorial Library & NSW & Pentecostal \\
\hline $\mathrm{C}$ & Australian Catholic University & AUST & Catholic \\
\hline A & Australian Lutheran College - Lohe Memorial Library & SA & Lutheran \\
\hline$A$ & Benedictine Community New Norcia - New Norcia Library & WA & Catholic \\
\hline$A$ & Bible College of South Australia & SA & Well-spread over all denominations \\
\hline A & Booth College of Mission - The Salvation Army & NZ & Salvation Army \\
\hline A & Booth College - Booth College Library & NSW & Salvation Army \\
\hline$A$ & Camden Theological Library - Uniting Church in Australia & NSW & Protestant \\
\hline $\mathrm{C}$ & Campion College - Campion College Library & NSW & Catholic \\
\hline A & Carey Baptist College - Ayson Clifford Library & NZ & Protestant / Baptist \\
\hline \multirow[t]{2}{*}{$B$} & Catholic Education Office - Catholic Resource \& Information Service & SA & Catholic \\
\hline & Catholic Education Office of WA - Catholic Library of WA & WA & Catholic \\
\hline A & Catholic Institute of Sydney - Veech Library & NSW & Catholic \\
\hline A & Catholic Theological College - Mannix Library & VIC & Catholic \\
\hline A & Christ College - Christ College Library & NSW & Presbyterian \\
\hline C & Christian Heritage College - Christian Heritage College Library & QLD & Well-spread over all denominations \\
\hline A & Divine World Missionaries - Patrick Murphy Memorial Library & VIC & Catholic \\
\hline $\mathrm{C}$ & Excelsia College - Gordon Moyes Library (formerly Wesley Institute) & NSW & Well-spread over all denominations \\
\hline A & Good Shepherd College - Colin Library & NZ & Catholic \\
\hline A & Harvest Bible College - QLD Library & QLD & Pentecostal \\
\hline A & Harvest Bible College - VIC Library & VIC & Pentecostal \\
\hline$A$ & HarvestWest Bible College - Jenni Roche Memorial Library & WA & Pentecostal \\
\hline A & $\begin{array}{l}\text { John Kinder Theological Library - Anglican Church in Aotearoa New Zealand and } \\
\text { Polynesia }\end{array}$ & NZ & Anglican / Methodist \\
\hline C & Lifeway College - David Yaxley Memorial Library & NZ & Well-spread over all denominations \\
\hline A & Malyon College (QLD Baptost College of Ministries) - Malyon College Library & QLD & Baptist \\
\hline A & Mary Andrews College & NSW & Anglican \\
\hline A & Morling College - Gilbert Wright Library & NSW & Baptist \\
\hline A & Nazarene Theological College - John D. Fulton Library & QLD & Methodist \\
\hline \multirow[t]{2}{*}{ A } & Presbyterian Research Centre - Hewitson Library & NZ & Well-spread over all denominations \\
\hline & Queensland Theological College - Gibson-Radclife Library & QLD & Presbyterian \\
\hline A & Ridley College - Leon Morris Library & VIC & Anglican \\
\hline A & St Athanasius Coptic Orthodox Theological College - Library & VIC & Orthodox \\
\hline$B$ & St Benedict's Monastery - St Benedicts Monastery Library & NSW & Cathoic \\
\hline \multirow[t]{3}{*}{ A } & St Francis Theological College - Roscoe Library & QLD & Anglican \\
\hline & St Mark's Anglican National Memorial Library - Charles Sturt University & ACT & Anglican \\
\hline & St Paschal Library - Franciscan Order of Friars Minor & $\mathrm{VIC}$ & Catholic \\
\hline A & Sunshine Coast Theological College & QLD & Presbyterian \\
\hline A & Sydney Missionary \& Bible College - Kerr Library & NSW & Well-spread over all denominations \\
\hline C & - Tabor Library & TAS & Protestant \\
\hline \multirow[t]{2}{*}{ C } & Tabor College Victoria & $\mathrm{VIC}$ & Well-spread over all denominations \\
\hline & Trinity College - Leeper \& Mollison Libraries & VIC & Anglican \\
\hline A & Triniy College - Trinity Theological Library & QLD & Protestant \\
\hline A & Trinity Theological College & WA & Well-spread over all denominations \\
\hline A & Vianney College - St Ann's Library & NSW & Catholic \\
\hline$A$ & Vose Seminary - Vose Library & WA & Baptist \\
\hline$A$ & Youthworks College - Youthworks College Library & NSW & Anglican / Presbyterian \\
\hline
\end{tabular}




\begin{tabular}{|l|c|l|c|}
\hline Anglican & 7 & Orthodox & 1 \\
\hline Baptist & 4 & Pentecostal & 4 \\
\hline Catholic & 12 & Presbyterian & 4 \\
\hline Lutheran & 1 & Protestant & 4 \\
\hline Methodist & 2 & Salvation Army & 2 \\
\hline
\end{tabular}

\section{Predominant Denomination of Collections 2014}

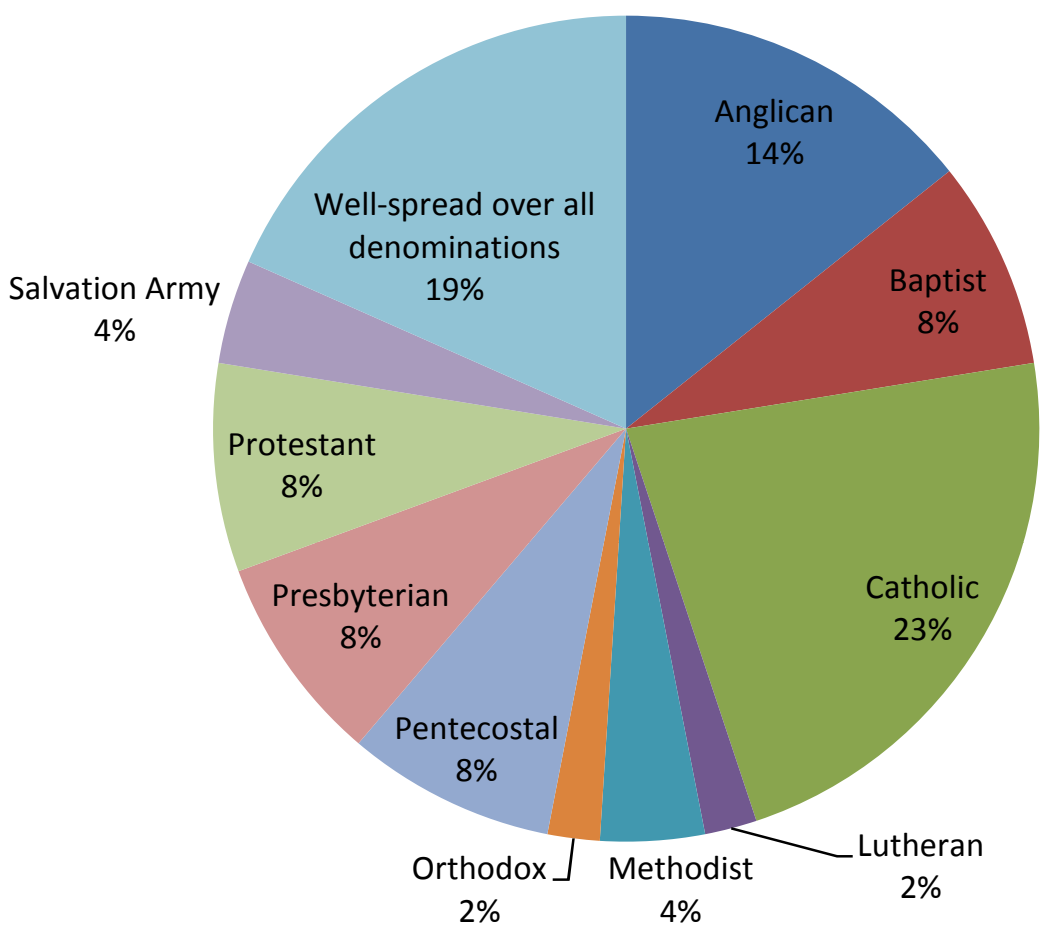


TABLE 22014 LIBRARY STAFF

\begin{tabular}{|c|c|c|c|c|c|c|c|c|}
\hline \multirow[t]{2}{*}{$\begin{array}{l}0 \\
0 \\
\ll\end{array}$} & \multirow[t]{2}{*}{ INSTITUTION } & \multirow[t]{2}{*}{$\begin{array}{c}\text { STATE/N } \\
z\end{array}$} & \multirow{2}{*}{$\begin{array}{c}\text { PROF. } \\
\text { POSITIONS } \\
{[1]}\end{array}$} & \multirow{2}{*}{$\begin{array}{c}\text { PARA-PROF. } \\
\text { POSITIONS } \\
{[2]} \\
\end{array}$} & \multirow{2}{*}{\begin{tabular}{|c|}
$\begin{array}{c}\text { SUPPORT } \\
\text { STAFF } \\
\text { POSITIONS }\end{array}$ \\
{$[3]$} \\
\end{tabular}} & \multirow{2}{*}{$\begin{array}{c}\text { VOLUNTEERS } \\
{[4]}\end{array}$} & \multirow{2}{*}{\begin{tabular}{|c|}
$\begin{array}{c}\text { TOTAL STAFF } \\
\text { Q1-Q4 }\end{array}$ \\
{$[5]$} \\
\end{tabular}} & \multirow{2}{*}{\begin{tabular}{|c} 
TOTAL STAFF \\
\# of People
\end{tabular}} \\
\hline & & & & & & & & \\
\hline A & Adelaide College of Divinity - Adelaide Theological Library & SA & 1.1 & 0.7 & & 0.15 & 1.95 & \\
\hline $\mathrm{C}$ & Alphacrucis College - James Wallace Memorial Library & NSW & 1 & & 0.45 & 0.33 & 1.78 & \\
\hline $\mathrm{C}$ & Australian Catholic University & AUST & 45.6 & 30.6 & 18.1 & & 94.30 & 110 \\
\hline A & Australian Lutheran College - Lohe Memorial Library & SA & 3.35 & & 0.4 & 3 & 6.75 & \\
\hline A & Benedictine Community New Norcia - New Norcia Library & WA & 0.8 & & & 0.025 & 0.83 & \\
\hline A & Bible College of South Australia & SA & 0.4 & & & 0.3 & 0.70 & 4 \\
\hline A & Booth College of Mission - The Salvation Army & $\mathrm{NZ}$ & 1 & & & & 1.00 & 1 \\
\hline A & Booth College - Booth College Library & NSW & 2 & & & & 2.00 & \\
\hline A & Camden Theological Library - Uniting Church in Australia & NSW & 1 & 1.25 & 0.75 & & 3.00 & \\
\hline $\mathrm{C}$ & Campion College - Campion College Library & NSW & 1 & & 4 & & 5.00 & 5 \\
\hline A & Carey Baptist College - Ayson Clifford Library & $\mathrm{NZ}$ & 2 & 1 & & & 3.00 & \\
\hline \multirow[t]{2}{*}{$B$} & $\begin{array}{l}\text { Catholic Education Office - Catholic Resource \& Information } \\
\text { Service }\end{array}$ & SA & 1 & 1 & & 0.1 & 2.10 & 3 \\
\hline & Catholic Education Office of WA - Catholic Library of WA & WA & 3 & 2 & 2 & & 7.00 & 7 \\
\hline A & Catholic Institute of Sydney - Veech Library & NSW & & & & & 0.00 & \\
\hline $\mathrm{A}$ & Catholic Theological College - Mannix Library & $\mathrm{VIC}$ & 2 & 1 & 0.3 & & 3.30 & \\
\hline A & Christ College - Christ College Library & NSW & 1 & & & & 1.00 & 1 \\
\hline $\mathrm{C}$ & Christian Heritage College - Christian Heritage College & QLD & 1 & 1 & 1.66 & & 3.66 & \\
\hline A & Divine World Missionaries - Patrick Murphy Memorial & $\mathrm{VIC}$ & 0.33 & & & & 0.33 & 1 \\
\hline$C$ & $\begin{array}{l}\text { Excelsia College - Gordon Moyes Library (formerly Wesley } \\
\text { Institute) }\end{array}$ & NSW & 2 & & & & 2.00 & 4 \\
\hline A & Good Shepherd College - Colin Library & $\mathrm{NZ}$ & 1 & & 0.25 & & 1.25 & 3 \\
\hline $\mathrm{A}$ & Harvest Bible College - QLD Library & QLD & 0.2 & & & & 0.20 & 1 \\
\hline A & Harvest Bible College - VIC Library & $\mathrm{VIC}$ & 1 & & & & 1.00 & 1 \\
\hline A & HarvestWest Bible College - Jenni Roche Memorial Library & WA & & & & & 0.00 & 2 \\
\hline A & $\begin{array}{l}\text { John Kinder Theological Library - Anglican Church in Aotearoa } \\
\text { New Zealand and Polynesia }\end{array}$ & NZ & & & & & 0.00 & 9 \\
\hline C & Lifeway College - David Yaxley Memorial Library & NZ & 0.7 & & & & 0.70 & \\
\hline $\mathrm{A}$ & $\begin{array}{l}\text { Malyon College (QLD Baptost College of Ministries) - } \\
\text { Malyon College Library }\end{array}$ & QLD & 0.95 & & & 0.3 & 1.25 & 5 \\
\hline A & Mary Andrews College & NSW & & & 0.33 & & 0.33 & 1 \\
\hline A & Morling College - Gilbert Wright Library & NSW & 1.2 & 0.84 & 0.35 & 0.14 & 2.53 & \\
\hline $\mathrm{A}$ & Nazarene Theological College - John D. Fulton Library & QLD & 1 & & 1 & & 2.00 & 2 \\
\hline \multirow[t]{2}{*}{ A } & Presbyterian Research Centre - Hewitson Library & NZ & 2 & 0.5 & & 0.5 & 3.00 & 3 \\
\hline & Queensland Theological College - Gibson-Radclife Library & QLD & 1 & & 0.24 & 0.9 & 2.14 & 3 \\
\hline $\mathrm{A}$ & Ridley College - Leon Morris Library & VIC & 1.8 & & 0.4 & & 2.20 & \\
\hline A & St Athanasius Coptic Orthodox Theological College - & $\mathrm{VIC}$ & 0.55 & & & & 0.55 & 1 \\
\hline $\mathrm{B}$ & St Benedict's Monastery - St Benedicts Monastery Library & NSW & 0.2 & & & & 0.20 & \\
\hline \multirow[t]{3}{*}{$A$} & St Francis Theological College - Roscoe Library & QLD & 1.4 & 0.37 & & 0.16 & 1.93 & \\
\hline & $\begin{array}{l}\text { St Mark's Anglican National Memorial Library - Charles } \\
\text { Sturt University }\end{array}$ & ACT & 2 & 2 & & 6 & 10.00 & \\
\hline & St Paschal Library - Franciscan Order of Friars Minor & VIC & 1.8 & & 0.6 & & 2.40 & \\
\hline A & Sunshine Coast Theological College & QLD & & 0.2 & & & 0.20 & 1 \\
\hline A & Sydney Missionary \& Bible College - Kerr Library & NSW & 1.6 & 1 & 0.66 & 0.37 & 3.63 & \\
\hline$C$ & Tabor College Tasmania - Tabor Library & TAS & 1 & & & 2 & 3.00 & 2 \\
\hline \multirow[t]{2}{*}{ C } & Tabor College Victoria & VIC & 2 & 0.5 & 0.6 & & 3.10 & \\
\hline & Trinity College - Leeper \& Mollison Libraries & VIC & 1.8 & 0.6 & 0.6 & & 3.00 & 6 \\
\hline $\mathrm{A}$ & Triniy College - Trinity Theological Library & QLD & & & & & 0.00 & 3 \\
\hline A & Trinity Theological College & WA & 1 & 0.6 & 0.11 & 0.2 & 1.91 & 5 \\
\hline A & Vianney College - St Ann's Library & NSW & 0.2 & & 1 & & 1.20 & \\
\hline A & Vose Seminary - Vose Library & WA & 1.2 & & 0.4 & 2.1 & 3.70 & \\
\hline A & Youthworks College - Youthworks College Library & NSW & & & 1 & 0.1 & 1.10 & 2 \\
\hline \multicolumn{3}{|c|}{ TOTAL } & 95.18 & 45.16 & 35.2 & 16.675 & 192.215 & 186 \\
\hline \multicolumn{3}{|c|}{ AVERAGE } & 2.3795 & 2.6564706 & 1.6 & 0.98088235 & 4.0896809 & 7 \\
\hline
\end{tabular}


TABLE 32014 LIBRARY SERVICES

\begin{tabular}{|c|c|c|c|c|c|c|c|c|c|}
\hline \multirow[t]{2}{*}{$\begin{array}{l}0 \\
m \\
<\end{array}$} & \multirow[t]{2}{*}{ INSTITUTION } & \multirow[t]{2}{*}{$\begin{array}{c}\text { STATE/N } \\
z\end{array}$} & $\begin{array}{c}\text { TOTAL } \\
\text { LOANS } 2014\end{array}$ & $\begin{array}{c}\text { ORIGINAL } \\
\text { LENT }\end{array}$ & $\begin{array}{l}\text { ILLS SUPPLIED } \\
\text { COPYIFAX } \\
\text { SUPPLIED }\end{array}$ & $\begin{array}{c}\text { TOTAL } \\
\text { SUPPLIED }\end{array}$ & $\begin{array}{l}\text { ITEMS } \\
\text { REQ'D }\end{array}$ & $\begin{array}{l}\text { LLS REQUESTEL } \\
\begin{array}{c}\text { COPY/FAX } \\
\text { REQ'D }\end{array}\end{array}$ & $\begin{array}{l}\text { TOTAL } \\
\text { REQ'D }\end{array}$ \\
\hline & & & [6] & [7] & [8] & [9] & [10] & [11] & [12] \\
\hline A & Adelaide College of Divinity - Adelaide Theological Library & SA & 10,163 & 206 & 102 & 308 & 8 & & 8 \\
\hline $\mathrm{C}$ & Alphacrucis College - James Wallace Memorial Library & NSW & 4,114 & 14 & & 14 & 2 & & 2 \\
\hline C & Australian Catholic University & AUST & 188,714 & 1,182 & 1,721 & 2,903 & 1,414 & 1,814 & 3,228 \\
\hline A & Benedictine Community New Norcia - New Norcia Library & WA & & 2 & 8 & 10 & 1 & & 1 \\
\hline A & Bible College of South Australia & SA & & & & 0 & & & 0 \\
\hline A & Booth College of Mission - The Salvation Army & NZ & 2,456 & & 2 & 2 & 6 & & 6 \\
\hline A & Booth College - Booth College Library & NSW & 2,343 & 5 & 10 & 15 & 10 & 5 & 15 \\
\hline A & Camden Theological Library - Uniting Church in Australia & NSW & 5,643 & 19 & 3 & 22 & 3 & 3 & 6 \\
\hline & Catholic Education Office of WA - Catholic Library of WA & WA & 11,104 & & & & & & \\
\hline A & Catholic Institute of Sydney - Veech Library & NSW & & & & 0 & & & 0 \\
\hline A & Catholic Theological College - Mannix Library & VIC & 17,282 & 17 & 7 & 24 & 11 & 14 & 25 \\
\hline A & Christ College - Christ College Library & NSW & 6,364 & & & 0 & 1 & 10 & 11 \\
\hline C & Christian Heritage College - Christian Heritage College & QLD & 23,237 & 1 & 1 & 2 & 7 & 6 & 13 \\
\hline A & Divine World Missionaries - Patrick Murphy Memorial & VIC & 200 & 10 & 5 & 15 & 1 & 3 & 4 \\
\hline C & $\begin{array}{l}\text { Excelsia College - Gordon Moyes Library (formerly Wesley } \\
\text { Institute) }\end{array}$ & NSW & 3,942 & & & 0 & 3 & 24 & 27 \\
\hline A & Good Shepherd College - Colin Library & NZ & & 48 & 10 & 58 & 62 & 10 & 1 \\
\hline A & Harvest Bible College - QLD Library & QLD & 518 & & & 0 & & & 0 \\
\hline A & Mary Andrews College & NSW & 1,647 & & & 0 & & & 0 \\
\hline A & Morling College - Gilbert Wright Library & NSW & 17,920 & & & 0 & & & 0 \\
\hline A & Nazarene Theological College - John D. Fulton Library & QLD & 4,199 & 9 & 2 & 11 & 4 & & 4 \\
\hline A & Presbyterian Research Centre - Hewitson Library & NZ & 2,830 & & & 123 & & & 5 \\
\hline & Queensland Theological College - Gibson-Radclife Library & QLD & 5,388 & 1 & 1 & 2 & 30 & 15 & 45 \\
\hline A & Ridley College - Leon Morris Library & VIC & 11,174 & & 3 & 3 & 5 & 14 & 19 \\
\hline A & St Athanasius Coptic Orthodox Theological College - & VIC & 287 & & & 0 & & 1 & 1 \\
\hline$B$ & St Benedict's Monastery - St Benedicts Monastery Library & NSW & & & & 0 & & & 0 \\
\hline A & St Francis Theological College - Roscoe Library & QLD & & & 6 & 6 & & & 0 \\
\hline & $\begin{array}{l}\text { St Mark's Anglican National Memorial Library - Charles } \\
\text { Sturt University }\end{array}$ & ACT & 9,359 & 46 & 561 & 607 & 30 & 10 & 40 \\
\hline & St Paschal Library - Franciscan Order of Friars Minor & VIC & 4,032 & 2 & 5 & 7 & 4 & 7 & 11 \\
\hline A & Sunshine Coast Theological College & QLD & 215 & & & 0 & & & 0 \\
\hline A & Sydney Missionary \& Bible College - Kerr Library & NSW & 13,300 & 2 & 1 & 3 & 3 & 6 & 9 \\
\hline C & Tabor College Tasmania - Tabor Library & TAS & 180 & & & 0 & & & 0 \\
\hline C & Tabor College Victoria & VIC & 5,609 & 1 & & 1 & & & 0 \\
\hline & Trinity College - Leeper \& Mollison Libraries & VIC & 14,952 & 4 & & 4 & & & 0 \\
\hline A & Triniy College - Trinity Theological Library & QLD & 2,333 & 7 & 3 & 10 & 19 & 82 & 101 \\
\hline $\mathrm{A}$ & Trinity Theological College & WA & 2,340 & 9 & 3 & 12 & 5 & 3 & 8 \\
\hline
\end{tabular}




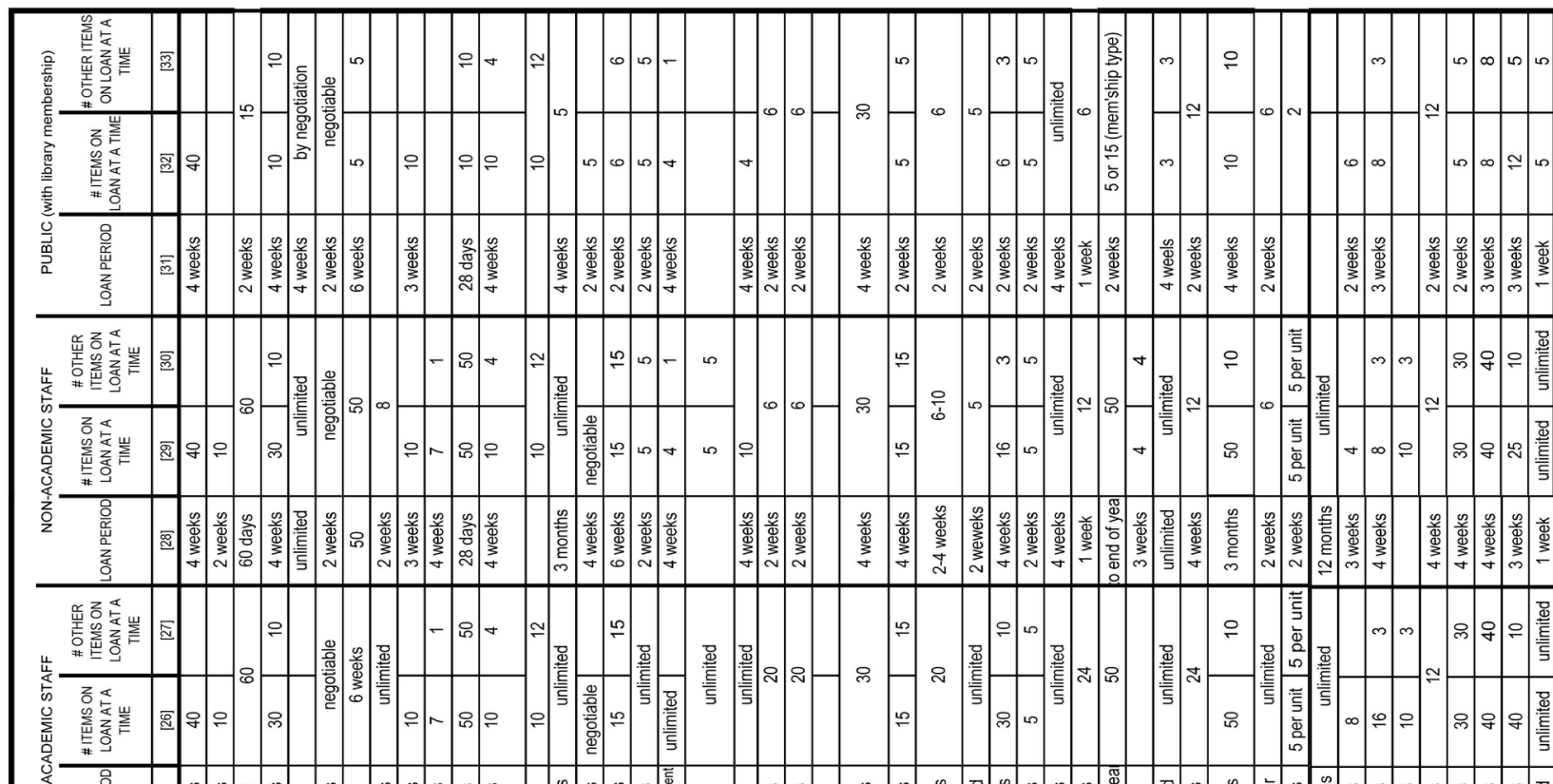

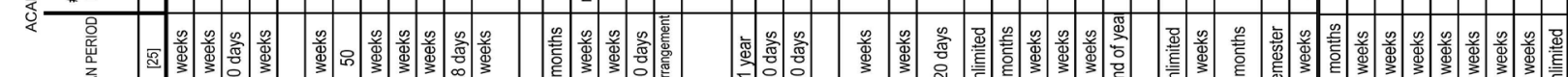

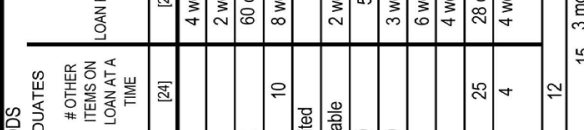

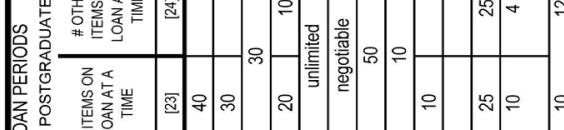

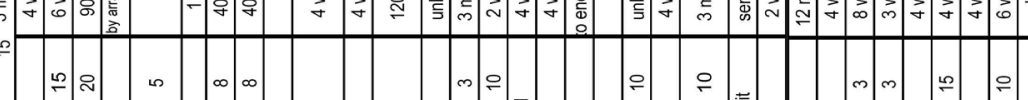

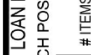

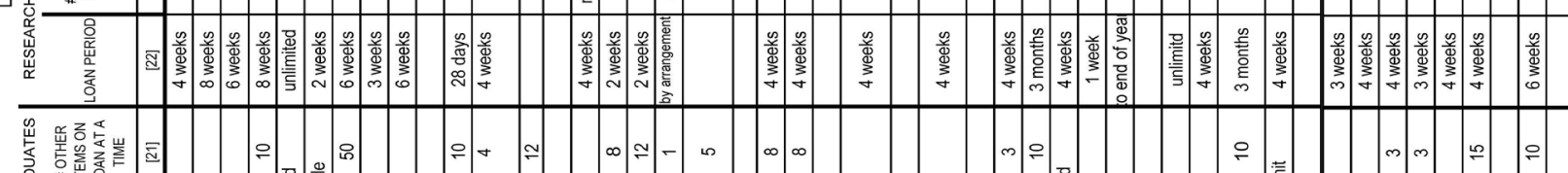

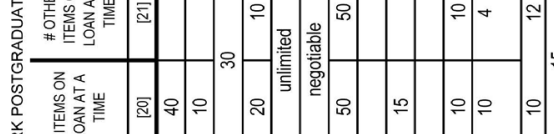

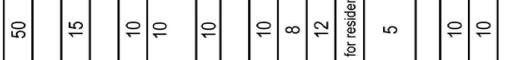

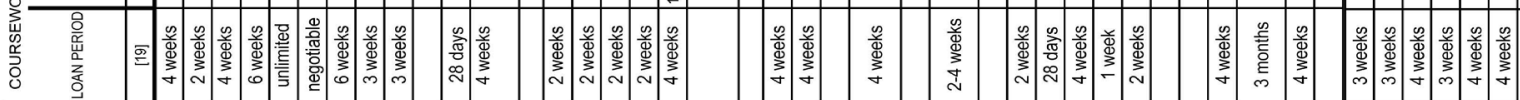

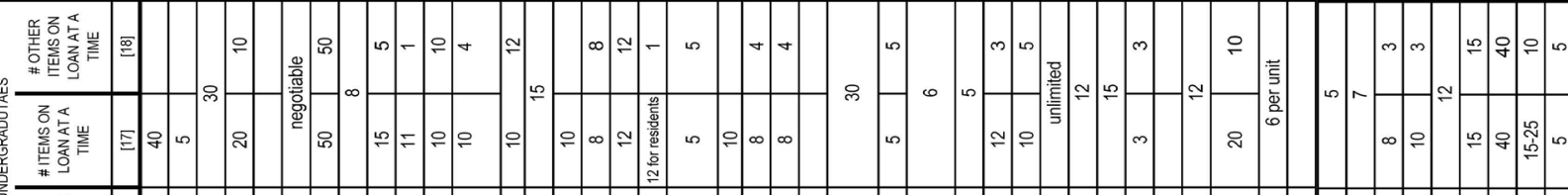

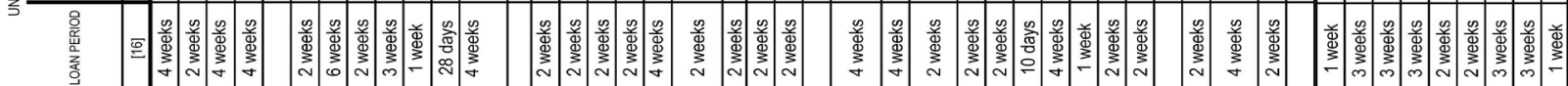

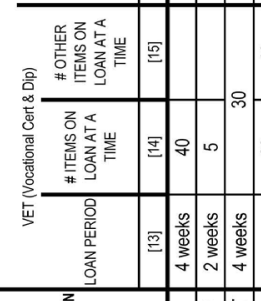

酸

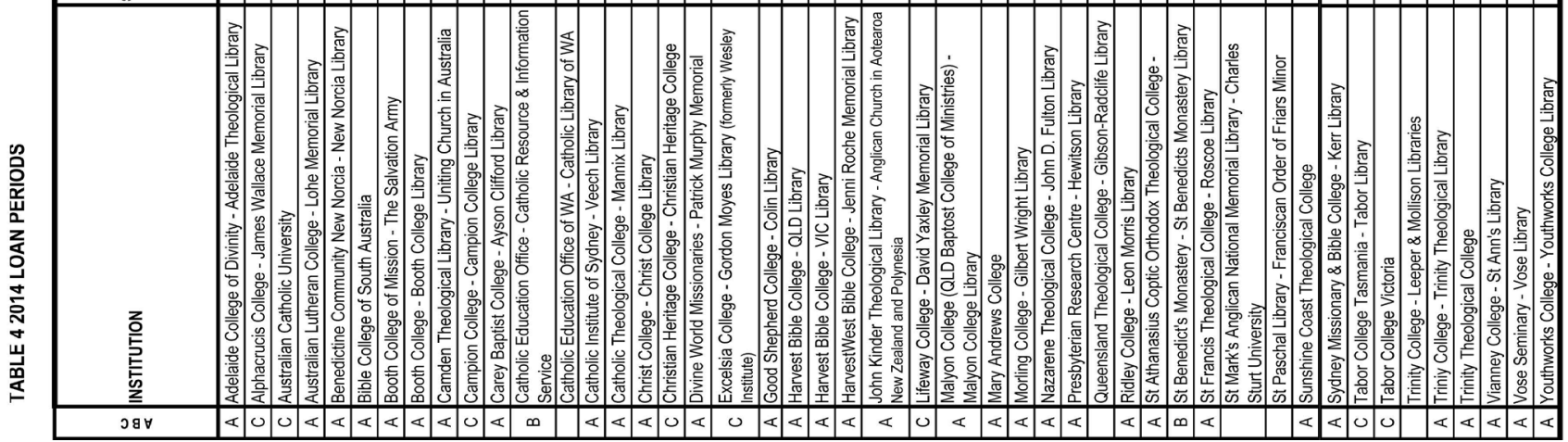


TABLE 52014 COLLECTION RESOURCES

\begin{tabular}{|c|c|c|c|c|c|c|c|c|c|c|c|c|c|c|}
\hline \multirow{3}{*}{$\begin{array}{l}0 \\
m \\
\alpha\end{array}$} & \multirow{3}{*}{ INSTITUTION } & \multirow{3}{*}{$\begin{array}{c}\text { STATE } / \mathbb{N} \\
z\end{array}$} & \multicolumn{3}{|c|}{ MONOGRAPH VOLUMES } & \multicolumn{3}{|c|}{ NON-BOOK MATERIALS } & \multirow{2}{*}{\begin{tabular}{|c|} 
TOAL SIZE \\
of COLL'N \\
2014 \\
\end{tabular}} & \multicolumn{3}{|c|}{ SERIAL VOLUMES } & \multirow[b]{2}{*}{$\begin{array}{l}\text { ELEC. } \\
\text { SERIALS }\end{array}$} & \multirow[b]{2}{*}{$\begin{array}{l}\text { ELECT. } \\
\text { D'BASES }\end{array}$} \\
\hline & & & ADDED 2014 & $\begin{array}{c}\text { DONATED } \\
2014\end{array}$ & \begin{tabular}{|c|} 
TOTAL END \\
2014
\end{tabular} & ADDED 2014 & $\begin{array}{c}\text { EBOOKS } \\
\text { ADDED } 2014\end{array}$ & $\begin{array}{c}\text { TOTAL END } \\
2014\end{array}$ & & $\begin{array}{c}\text { NEW SUBS } \\
2014\end{array}$ & $\begin{array}{c}\text { CURRENT } \\
\text { SUBS }\end{array}$ & $\begin{array}{c}\text { TOTAL END } \\
2013\end{array}$ & & \\
\hline & & & [34] & [35] & {$[36]$} & [37] & {$[38]$} & [39] & [40] & [41] & [42] & [43] & [44] & [45] \\
\hline A & Adelaide College of Divinity - Adelaide Theological Library & SA & 923 & 416 & & & 130 & & 71,144 & 2 & 204 & & 32 & 13 \\
\hline C & Alphacrucis College - James Wallace Memorial Library & NSW & 4,500 & 700 & 50,402 & & & & & & & & & 9 \\
\hline C & Australian Catholic University & AUST & 10,370 & & 417,543 & & 25,369 & 358,072 & 775,615 & 3 & 375 & 657 & 12,941 & 560 \\
\hline $\mathrm{A}$ & Australian Lutheran College - Lohe Memorial Library & $\mathrm{SA}$ & 1,044 & 71 & 87,656 & 24 & 8 & 2,177 & 89,833 & 1 & 190 & 720 & & 6 \\
\hline A & Benedictine Community New Norcia - New Norcia Library & WA & 635 & & 81,000 & 5 & & 5 & 81,005 & & & & & \\
\hline A & Bible College of South Australia & SA & 197 & 405 & 24,000 & 2 & & 208 & 24,300 & 1 & 35 & 431 & 13 & 3 \\
\hline A & Booth College of Mission - The Salvation Army & $\mathrm{NZ}$ & 411 & & & 39 & & & 18,809 & & 47 & & & 3 \\
\hline A & Booth College - Booth College Library & NSW & 1,084 & 20 & 24,502 & 50 & 10 & 60 & 24,562 & 6 & 51 & 123 & & 4 \\
\hline A & Camden Theological Library - Uniting Church in Australia & NSW & 2,337 & & 70,640 & & & & 70,640 & & 70 & 562 & & 4 \\
\hline C & Campion College - Campion College Library & NSW & & & & & & & 0 & & & & & \\
\hline A & Carey Baptist College - Ayson Clifford Library & $\mathrm{NZ}$ & 2,156 & & & & 73 & & 0 & 2 & & & 15 & 5 \\
\hline B & $\begin{array}{l}\text { Catholic Education Office - Catholic Resource \& Information } \\
\text { Service }\end{array}$ & SA & 893 & 39 & 22,916 & 87 & & 2,135 & 25,051 & 2 & 44 & 46 & 1 & \\
\hline & Catholic Education Office of WA - Catholic Library of WA & WA & 410 & 42 & 9,313 & 93 & 384 & 4,315 & & & 52 & 52 & 2 & 9 \\
\hline A & Catholic Institute of Sydney - Veech Library & NSW & & & & & & & 0 & & & & & \\
\hline A & Catholic Theological College - Mannix Library & VIC & 627 & 444 & 132,071 & & & 88 & 132,159 & 3 & 192 & 498 & 27 & 12 \\
\hline A & Christ College - Christ College Library & NSW & 953 & 338 & 42,818 & 4 & & 909 & 43,727 & & 52 & 299 & & 3 \\
\hline $\mathrm{C}$ & Christian Heritage College - Christian Heritage College & QLD & 1,718 & & 52,417 & 12 & 44 & 566 & 52,983 & & 68 & 274 & 1 & 32 \\
\hline A & Divine World Missionaries - Patrick Murphy Memorial & VIC & 150 & 100 & 200 & 10 & & 10 & 210 & & 30 & 30 & & \\
\hline c & $\begin{array}{l}\text { Excelsia College - Gordon Moyes Library (formerly Wesley } \\
\text { Institute) }\end{array}$ & NSW & 400 & & 34,000 & 20 & & & 34,000 & & 5 & 300 & & 10 \\
\hline A & Good Shepherd College - Colin Library & $\mathrm{NZ}$ & 751 & 40 & 100,000 & & 27 & & 100,000 & & 91 & 91 & & 1 \\
\hline A & Harvest Bible College - QLD Library & QLD & & & 15,504 & & & 1,963 & 17,466 & & & & 15 & 4 \\
\hline A & Harvest Bible College - VIC Library & VIC & & & 38,082 & & & 7,110 & 45,192 & & & & 15 & 4 \\
\hline A & HarvestWest Bible College - Jenni Roche Memorial Library & WA & 29 & 401 & 13,965 & 3 & & 428 & 14,393 & & 1 & & & \\
\hline A & $\begin{array}{l}\text { John Kinder Theological Library - Anglican Church in Aotearoa } \\
\text { New Zealand and Polynesia }\end{array}$ & NZ & 1,750 & 900 & 100,540 & & 107 & & 100,647 & & & & & 8 \\
\hline $\mathrm{C}$ & Lifeway College - David Yaxley Memorial Library & NZ & 19 & 140 & 6,237 & 9 & 5 & 207 & 6,444 & 3 & 15 & 44 & 2 & 1 \\
\hline A & $\begin{array}{l}\text { Malyon College (QLD Baptost College of Ministries) - } \\
\text { Malyon College Library }\end{array}$ & QLD & 1,054 & 366 & 25,679 & 7 & 106 & 1,822 & 27,501 & 6 & 89 & 236 & & 2 \\
\hline A & Mary Andrews College & NSW & 356 & 39 & 67,000 & 15 & & 129 & 6,829 & & 19 & 25 & & \\
\hline A & Morling College - Gilbert Wright Library & NSW & 779 & 256 & & 79 & 2 & 81 & 56,518 & & 280 & 280 & 73 & 2 \\
\hline A & Nazarene Theological College - John D. Fulton Library & QLD & 178 & 40 & 32,994 & 20 & 500 & 520 & 33,514 & 2 & 57 & 95 & & 1 \\
\hline A & Presbyterian Research Centre - Hewitson Library & NZ & 79 & 746 & & 57 & & 57 & & & 28 & 889 & & 3 \\
\hline & Queensland Theological College - Gibson-Radclife Library & QLD & 886 & 78 & 19,260 & 35 & 1,900 & 3,161 & 22,421 & & 37 & 89 & 6 & 7 \\
\hline A & Ridley College - Leon Morris Library & VIC & 1,396 & & 55,284 & 4 & 11 & 793 & 56,077 & 5 & 153 & 427 & & 6 \\
\hline A & St Athanasius Coptic Orthodox Theological College - & $\mathrm{VIC}$ & 89 & 231 & 3,815 & 7 & 37 & 44 & 3,859 & 1 & 1 & 1 & & 3 \\
\hline $\mathrm{B}$ & St Benedict's Monastery - St Benedicts Monastery Library & NSW & 202 & 25 & 20,435 & 29 & & 265 & 20,700 & & 45 & 145 & & \\
\hline A & St Francis Theological College - Roscoe Library & QLD & 1,090 & 495 & 21,900 & 4 & 61 & & 21,900 & 1 & 48 & 48 & 1 & \\
\hline & $\begin{array}{l}\text { St Mark's Anglican National Memorial Library - Charles } \\
\text { Sturt University }\end{array}$ & ACT & 783 & 854 & 64,939 & 30 & 16 & 548 & 65,487 & 3 & 95 & 401 & 5 & 8 \\
\hline & St Paschal Library - Franciscan Order of Friars Minor & $\mathrm{VIC}$ & 1,256 & 143 & 61,969 & 4 & & 138 & 62,107 & 1 & 149 & 343 & 2 & 5 \\
\hline A & Sunshine Coast Theological College & QLD & 314 & 15 & 3,606 & & & 100 & 3,706 & & 15 & 36 & 1 & 2 \\
\hline A & Sydney Missionary \& Bible College - Kerr Library & NSW & 1,057 & 220 & 40,143 & 7 & 600 & 803 & 40,946 & 6 & 140 & 263 & 22 & 7 \\
\hline $\mathrm{C}$ & Tabor College Tasmania - Tabor Library & TAS & & & 10,410 & 81 & & 255 & 10,665 & 17 & 3 & & & \\
\hline C & Tabor College Victoria & $\mathrm{VIC}$ & 968 & 1,092 & 61,010 & 62 & 1 & 3,753 & 64,763 & 1 & 90 & 860 & 3 & 6 \\
\hline & Trinity College - Leeper \& Mollison Libraries & VIC & 568 & 12 & 30,641 & 15 & 152 & 12,505 & 43,146 & 2 & 30 & 32 & 1 & 3 \\
\hline A & Triniy College - Trinity Theological Library & QLD & 522 & 278 & 25,166 & 26 & 105 & 215 & 25,381 & 1 & 18 & 279 & 14 & 1 \\
\hline $\mathrm{A}$ & Trinity Theological College & WA & 812 & 640 & 25,280 & 17 & 5 & 39 & 25,319 & & 58 & 128 & & 10 \\
\hline $\mathrm{A}$ & Vianney College - St Ann's Library & NSW & & & 199 & & & 199 & 18,470 & & 48 & 48 & & 2 \\
\hline $\mathrm{A}$ & Vose Seminary - Vose Library & WA & 590 & 1,210 & 44,139 & 59 & 114 & 38,860 & 82,999 & & 80 & & & 4 \\
\hline A & Youthworks College - Youthworks College Library & NSW & 57 & & 4,893 & 3 & & 155 & 5,048 & & 15 & 56 & & \\
\hline & TAL & & 44,393 & 10,796 & $1,942,568$ & 919 & 29,767 & 442,695 & $2,425,536$ & 69 & 3,020 & 8,808 & 13,192 & 763 \\
\hline & RAGE & & 1,083 & 337 & 48,564 & 28 & 1,240 & 12,297 & 55,126 & & 77 & \begin{tabular}{r|}
259 \\
\end{tabular} & 628 & 21 \\
\hline
\end{tabular}




\begin{tabular}{|c|c|c|c|c|c|c|c|c|c|}
\hline \multirow[t]{2}{*}{$\begin{array}{ll}0 \\
m \\
\ll\end{array}$} & \multirow[t]{2}{*}{ INSTITUTION } & \multirow[t]{2}{*}{$\begin{array}{c}\text { STATE/N } \\
Z\end{array}$} & MONOGRAPHS & $\begin{array}{l}\text { NON-BOOK } \\
\text { MATERIALS }\end{array}$ & EBOOKS & SERIAL SUBS. & $\begin{array}{l}\text { ELECTRONIC SERIAL } \\
\text { SUBS. }\end{array}$ & $\begin{array}{c}\text { ELECTRONIC } \\
\text { DATABASE SUBS. }\end{array}$ & TOTAL SPENT \\
\hline & & & [46] & [47] & [48] & [49] & [50] & [51] & [52] \\
\hline A & Adelaide College of Divinity - Adelaide Theological Library & SA & \multicolumn{3}{|c|}{$\$ 40,335$} & \multicolumn{2}{|c|}{$\$ 30,774$} & $\$ 13,283$ & $\$ 84,392$ \\
\hline$C$ & Alphacrucis College - James Wallace Memorial Library & NSW & & & & & & & $\$ 0$ \\
\hline$C$ & Australian Catholic University & AUST & $\$ 803,006$ & & 1008114 & $\$ 64,304$ & & $\$ 3,360,790$ & $\$ 4,936,214$ \\
\hline A & Australian Lutheran College - Lohe Memorial Library & SA & $\$ 28,198$ & $\$ 712$ & $\$ 148$ & $\$ 12$ & 4,466 & $\$ 7,494$ & $\$ 51,018$ \\
\hline $\mathrm{A}$ & Benedictine Community New Norcia - New Norcia Library & WA & $\$ 7,953$ & $\$ 75$ & & $\$ 2,160$ & & & $\$ 10,188$ \\
\hline A & Bible College of South Australia & SA & & & & \multicolumn{3}{|c|}{$\$ 4,657$} & $\$ 10,810$ \\
\hline A & Booth College of Mission - The Salvation Army & $\mathrm{NZ}$ & $\$ 6,789$ & 962.38 & & $\$ 3,391$ & & & $\$ 11,142$ \\
\hline A & Booth College - Booth College Library & NSW & \multicolumn{3}{|c|}{$\$ 35,000$} & \multicolumn{3}{|c|}{$\$ 13,000$} & $\$ 48,000$ \\
\hline A & Camden Theological Library - Uniting Church in Australia & NSW & $\$ 35,000$ & & & $\$ 12,500$ & & $\$ 13,000$ & $\$ 60,500$ \\
\hline$C$ & Campion College - Campion College Library & NSW & & & & & & & $\$ 0$ \\
\hline A & Carey Baptist College - Ayson Clifford Library & NZ & $\$ 39,500$ & & $\$ 5,282$ & & & & $\$ 44,782$ \\
\hline \multirow[t]{2}{*}{ B } & $\begin{array}{l}\text { Catholic Education Office - Catholic Resource \& Information } \\
\text { Service }\end{array}$ & SA & & & & & & & $\$ 0$ \\
\hline & Catholic Education Office of WA - Catholic Library of WA & WA & $\$ 9,000$ & $\$ 6,000$ & $\$ 15,500$ & $\$ 13,000$ & $\$ 400$ & $\$ 38,800$ & $\$ 82,700$ \\
\hline A & Catholic Institute of Sydney - Veech Library & NSW & & & & & & & $\$ 0$ \\
\hline A & Catholic Theological College - Mannix Library & $\mathrm{VIC}$ & $\$ 48,400$ & & & $\$ 29,600$ & & $\$ 13,900$ & $\$ 91,900$ \\
\hline$A$ & Christ College - Christ College Library & NSW & & & & & & & $\$ 0$ \\
\hline$C$ & Christian Heritage College - Christian Heritage College & QLD & $\$ 47,638$ & $\$ 8,703$ & $\$ 3,136$ & $\$ 250$ & $\$ 577$ & $\$ 11,335$ & $\$ 71,639$ \\
\hline A & Divine World Missionaries - Patrick Murphy Memorial & VIC & $\$ 5,350$ & & & $\$ 2,100$ & & & $\$ 7,450$ \\
\hline C & $\begin{array}{l}\text { Excelsia College - Gordon Moyes Library (formerly Wesley } \\
\text { Institute) }\end{array}$ & NSW & & & & & & & $\$ 0$ \\
\hline $\mathrm{BC}$ & Good Shepherd Collehe - Colin Library & $\mathrm{NZ}$ & $\$ 30,000$ & & & $\$ 10,000$ & & & $\$ 40,000$ \\
\hline A & Harvest Bible College - QLD Library & QLD & & & & & & & $\$ 2,001$ \\
\hline A & Harvest Bible College - VIC Library & VIC & & & & & & & $\$ 26,359$ \\
\hline A & HarvestWest Bible College - Jenni Roche Memorial Library & WA & & & & & & $\$ 3,500$ & $\$ 3,500$ \\
\hline A & $\begin{array}{l}\text { John Kinder Theological Library - Anglican Church in Aotearoa } \\
\text { New Zealand and Polynesia }\end{array}$ & NZ & \multicolumn{2}{|c|}{$\$ 88,000$} & 7400 & \multicolumn{2}{|c|}{$\$ 26,000$} & $\$ 14,000$ & $\$ 135,400$ \\
\hline$C$ & Lifeway College - David Yaxley Memorial Library & NZ & $\$ 1,051$ & $\$ 204$ & & $\$ 1,899$ & $\$ 95$ & $\$ 60$ & $\$ 3,309$ \\
\hline A & $\begin{array}{l}\text { Malyon College (QLD Baptost College of Ministries) - } \\
\text { Malyon College Library }\end{array}$ & QLD & $\$ 38,500$ & $\$ 100$ & $\$ 4,600$ & $\$ 8,600$ & & $\$ 9,400$ & $\$ 61,200$ \\
\hline $\mathrm{A}$ & Mary Andrews College & NSW & & & & & & & $\$ 10,453$ \\
\hline A & Morling College - Gilbert Wright Library & NSW & $\$ 31$, & & & $\$ 10,655$ & $\$ 5,840$ & $\$ 27,000$ & $\$ 64,100$ \\
\hline A & Nazarene Theological College - John D. Fulton Library & QLD & $\$ 9,000$ & $\$ 150$ & $\$ 1,800$ & $\$ 8,000$ & & $\$ 1,500$ & $\$ 19,950$ \\
\hline \multirow[t]{2}{*}{ A } & Presbyterian Research Centre - Hewitson Library & NZ & $\$ 4,000$ & & & $\$ 2,000$ & & $\$ 2,078$ & $\$ 8,078$ \\
\hline & Queensland Theological College - Gibson-Radclife Library & QLD & $\$ 29,033$ & $\$ 78$ & $\$ 291$ & $\$ 3,817$ & \multicolumn{2}{|c|}{$\$ 8,922$} & $\$ 55,966$ \\
\hline A & Ridley College - Leon Morris Library & VIC & $\$ 51,160$ & & $\$ 3,474$ & $\$ 14,980$ & & $\$ 9,416$ & $\$ 79,029$ \\
\hline A & St Athanasius Coptic Orthodox Theological College - & VIC & $\$ 3,500$ & & & $\$ 80$ & & & $\$ 3,580$ \\
\hline $\mathrm{B}$ & St Benedict's Monastery - St Benedicts Monastery Library & NSW & $\$ 5,620$ & $\$ 200$ & & $\$ 3,800$ & & & $\$ 9,620$ \\
\hline \multirow{3}{*}{ A } & St Francis Theological College - Roscoe Library & QLD & $\$ 21,415$ & $\$ 87$ & $\$ 1,300$ & $\$ 7,645$ & $\$ 206$ & & $\$ 30,653$ \\
\hline & $\begin{array}{l}\text { St Mark's Anglican National Memorial Library - Charles } \\
\text { Sturt University }\end{array}$ & ACT & $\$ 38,000$ & & $\$ 1,700$ & $\$ 18,051$ & $\$ 1,039$ & $\$ 3,742$ & $\$ 41,742$ \\
\hline & St Paschal Library - Franciscan Order of Friars Minor & VIC & $\$ 4,900$ & $\$ 100$ & & $\$ 30,569$ & & $\$ 3,831$ & $\$ 43,231$ \\
\hline A & Sunshine Coast Theological College & QLD & $\$ 6,283$ & & & $\$ 1,050$ & & $\$ 7,997$ & $\$ 16,097$ \\
\hline A & Sydney Missionary \& Bible College - Kerr Library & NSW & $\$ 40,840$ & $\$ 532$ & $\$ 1,355$ & $\$ 7,982$ & $\$ 9,980$ & $\$ 7,834$ & $\$ 60,541$ \\
\hline$C$ & Tabor College Tasmania - Tabor Library & TAS & $\$ 2,894$ & & & $\$ 1,266$ & & & $\$ 4,160$ \\
\hline \multirow[t]{2}{*}{$\mathrm{C}$} & Tabor College Victoria & VIC & $\$ 38,378$ & & $\$ 347$ & $\$ 11,628$ & & $\$ 4,327$ & $\$ 54,681$ \\
\hline & Trinity College - Leeper \& Mollison Libraries & $\mathrm{VIC}$ & $\$ 32,321$ & $\$ 400$ & $\$ 11,558$ & $\$ 5,000$ & $\$ 180$ & $\$ 6,330$ & $\$ 55,789$ \\
\hline A & Triniy College - Trinity Theological Library & QLD & $\$ 19,564$ & $\$ 40$ & $\$ 4,064$ & \multicolumn{3}{|c|}{$\$ 3,278$} & $\$ 26,946$ \\
\hline$A$ & Trinity Theological College & WA & $\$ 39,206$ & $\$ 67$ & $\$ 213$ & $\$ 10,422$ & & $\$ 19,217$ & $\$ 69,125$ \\
\hline A & Vianney College - St Ann's Library & NSW & $\$ 3,777$ & & & $\$ 3,856$ & & $\$ 579$ & $\$ 8,212$ \\
\hline $\mathrm{A}$ & Vose Seminary - Vose Library & WA & $\$ 20,091$ & $\$ 979$ & $\$ 7,588$ & $\$ 5,500$ & & $\$ 9,454$ & $\$ 43,612$ \\
\hline A & Youthworks College - Youthworks College Library & NSW & & $\$ 60$ & & & & & $\$ 60$ \\
\hline TOT & TAL & & $\$ 1,664,964$ & $\$ 19,449$ & & $\$ 386,280$ & $\$ 27,239$ & $\$ 3,588,866$ & $\$ 6,488,128$ \\
\hline AVE & ERAGE & & $\$ 47,570$ & $\$ 1,080$ & & $\$ 11,037$ & $\$ 3,027$ & $\$ 149,536$ & $\$ 138,045$ \\
\hline
\end{tabular}

Note: NZ Libraries use NZ\$, all other figures are AUD\$ 
TABLE 72014 INSTITUTIONAL POPULATION

\begin{tabular}{|c|c|c|c|c|c|c|c|c|c|c|c|}
\hline \multirow{3}{*}{$\begin{array}{l}0 \\
\dot{m} \\
\&\end{array}$} & \multirow{3}{*}{ INSTITUTION } & \multirow{3}{*}{$\begin{array}{c}\text { STATE/N } \\
z\end{array}$} & \multicolumn{2}{|c|}{ ACADEMIC STAFF } & \multicolumn{2}{|c|}{ NON-ACADEMIC STAFF } & \multicolumn{4}{|c|}{ STUDENT POPULATION } & \multirow[b]{2}{*}{$\begin{array}{l}\text { REGISTERED } \\
\text { BORROWERS }\end{array}$} \\
\hline & & & FULL TIME & PART TIME & FULL TIME & PART TIME & FULL TIME & PART TIME & $\begin{array}{l}\text { TOTAL \# OF } \\
\text { STUDENTS }\end{array}$ & EFT & \\
\hline & & & [53] & [54] & [55] & [56] & [57] & [58] & [59] & [60] & [61] \\
\hline A & Adelaide College of Divinity - Adelaide Theological Library & SA & 6 & 40 & 4 & 8 & & & 280 & 89.100 & 368 \\
\hline C & Alphacrucis College - James Wallace Memorial Library & NSW & & & & & & & & & \\
\hline C & Australian Catholic University & AUST & & & & & & & & & \\
\hline A & Australian Lutheran College - Lohe Memorial Library & SA & 21 & 7 & 6 & 12 & 46 & 177 & 223 & 77.290 & 35 \\
\hline A & Benedictine Community New Norcia - New Norcia Library & WA & 1 & & & & & & & & \\
\hline A & Bible College of South Australia & SA & 5 & 2 & 2 & 2 & & & & $37.5 / 30.13$ & 8 \\
\hline A & Booth College of Mission - The Salvation Army & $\mathrm{NZ}$ & 11 & 2 & 8 & 5 & 29 & 2 & 31 & 29.375 & 392 \\
\hline A & Booth College - Booth College Library & NSW & 4 & 14 & 4 & 5 & 21 & 115 & 136 & 52.000 & 10 \\
\hline A & Camden Theological Library - Uniting Church in Australia & NSW & & & & & & & & & \\
\hline C & Campion College - Campion College Library & NSW & 5 & 7 & 6 & 4 & & & & & \\
\hline $\mathrm{A}$ & Carey Baptist College - Ayson Clifford Library & $\mathrm{NZ}$ & 8 & 6 & 9 & 5 & 210 & 140 & 350 & 135.000 & \\
\hline B & $\begin{array}{l}\text { Catholic Education Office - Catholic Resource \& Information } \\
\text { Service }\end{array}$ & SA & & & & & & & 1,052 & & 7,704 \\
\hline & Catholic Education Office of WA - Catholic Library of WA & WA & & & & & & & & & 1,944 \\
\hline $\mathrm{A}$ & Catholic Institute of Sydney - Veech Library & NSW & & & & & & & & & \\
\hline A & Catholic Theological College - Mannix Library & $\mathrm{VIC}$ & 6 & 51 & 4 & & 65 & 409 & 474 & 186.000 & 496 \\
\hline A & Christ College - Christ College Library & NSW & 5 & 5 & 2 & 2 & 72 & 264 & 336 & 132.300 & 80 \\
\hline $\mathrm{C}$ & Christian Heritage College - Christian Heritage College & QLD & & & & & & & & & \\
\hline A & Divine World Missionaries - Patrick Murphy Memorial & VIC & & 2 & & & 5 & 2 & 13 & & 95 \\
\hline C & $\begin{array}{l}\text { Excelsia College - Gordon Moyes Library (formerly Wesley } \\
\text { Institute) }\end{array}$ & NSW & & & & & & & & & \\
\hline $\mathrm{A}$ & Good Shepherd College - Colin Library & NZ & 10 & 6 & 2 & 3 & & & 63 & 27.000 & \\
\hline A & Harvest Bible College - QLD Library & QLD & 2 & & 2 & & & & & & \\
\hline A & Harvest Bible College - VIC Library & VIC & 9 & & 11 & & & & & & \\
\hline A & HarvestWest Bible College - Jenni Roche Memorial Library & WA & 2 & 4 & 1 & 2 & & & & & \\
\hline A & $\begin{array}{l}\text { John Kinder Theological Library - Anglican Church in Aotearoa } \\
\text { New Zealand and Polynesia }\end{array}$ & $\mathrm{NZ}$ & & & & & 60 & 69 & & & 900 \\
\hline C & Lifeway College - David Yaxley Memorial Library & NZ & & & & & & & & & \\
\hline A & $\begin{array}{l}\text { Malyon College (QLD Baptost College of Ministries) - } \\
\text { Malyon College Library }\end{array}$ & QLD & 7 & 5 & 2 & 4 & 73 & 160 & 233 & 117.000 & 3 \\
\hline A & Mary Andrews College & NSW & 1 & 8 & 1 & 4 & 2 & 241 & 243 & 44.750 & 14 \\
\hline A & Morling College - Gilbert Wright Library & NSW & 16 & 12 & 8 & 13 & & & 1,065 & 67.000 & \\
\hline A & Nazarene Theological College - John D. Fulton Library & QLD & 5 & 5 & 2 & 3 & 18 & 22 & 40 & 12.000 & 15 \\
\hline A & Presbyterian Research Centre - Hewitson Library & $\mathrm{NZ}$ & 3 & 1 & 1 & & & & & & 1,098 \\
\hline & Queensland Theological College - Gibson-Radclife Library & QLD & 7 & 7 & 1 & 4 & 68 & 50 & 129 & 79.500 & 699 \\
\hline A & Ridley College - Leon Morris Library & VIC & 9 & 9 & 3 & 14 & 61 & 180 & 241 & 112.250 & 120 \\
\hline A & St Athanasius Coptic Orthodox Theological College - & VIC & 1 & 5 & 1 & & & 64 & 64 & 12.130 & \\
\hline $\mathrm{B}$ & St Benedict's Monastery - St Benedicts Monastery Library & NSW & 6 & & & & & & & & 23 \\
\hline A & St Francis Theological College - Roscoe Library & QLD & 4 & 14 & 2 & & 10 & 110 & 120 & 65.000 & 540 \\
\hline & $\begin{array}{l}\text { St Mark's Anglican National Memorial Library - Charles } \\
\text { Sturt University }\end{array}$ & ACT & 10 & 30 & 3 & 5 & & & & & 879 \\
\hline & St Paschal Library - Franciscan Order of Friars Minor & VIC & 2 & 27 & 1 & 5 & 38 & 194 & 232 & 62.000 & 298 \\
\hline A & Sunshine Coast Theological College & QLD & 1 & 1 & 1 & 2 & & 5 & 5 & 2.000 & 6 \\
\hline A & Sydney Missionary \& Bible College - Kerr Library & NSW & 15 & 26 & 14 & 19 & 155 & 322 & 477 & 229.000 & \\
\hline C & Tabor College Tasmania - Tabor Library & TAS & & & & & & & & & \\
\hline $\mathrm{C}$ & Tabor College Victoria & VIC & 4 & 6 & 4 & 12 & 138 & 194 & 332 & 138.000 & 4,000 \\
\hline & Trinity College - Leeper \& Mollison Libraries & VIC & & & & & & & 1,032 & & 1,270 \\
\hline A & Triniy College - Trinity Theological Library & QLD & 7 & 5 & 1 & & 15 & 77 & 92 & 11.500 & 442 \\
\hline $\mathrm{A}$ & Trinity Theological College & WA & 5 & 2 & 4 & 10 & 26 & 55 & 81 & 42.875 & 116 \\
\hline A & Vianney College - St Ann's Library & NSW & 4 & 10 & 4 & 4 & 26 & & 26 & 21.610 & 92 \\
\hline A & Vose Seminary - Vose Library & WA & 7 & 12 & 4 & 3 & 59 & 120 & 179 & 71.900 & 56 \\
\hline A & Youthworks College - Youthworks College Library & NSW & 2 & 10 & 1 & 3 & & & & & 264 \\
\hline TO & TAL & & 210.5 & 341 & 119 & 153 & 1197 & 2972 & 7549 & 1816.580 & 21967 \\
\hline & ERAGE & & 6.1911765 & 11 & 3.71875 & 6.12 & 57 & 135.09091 & 279.59259 & 75.691 & 757.4827586 \\
\hline
\end{tabular}




\section{TABLE 82014 BIBLIOGRAPHIC INSTRUCTION}

\begin{tabular}{|c|c|c|c|c|}
\hline \multirow{3}{*}{$\begin{array}{ll}0 \\
0 \\
\dot{m}\end{array}$} & \multirow{3}{*}{ INSTITUTION } & \multirow{3}{*}{$\begin{array}{c}\text { STATE/N } \\
Z\end{array}$} & \multicolumn{2}{|c|}{ BIBLIOGRAPHIC INSTRUCTION } \\
\hline & & & $\begin{array}{c}\text { NO. OF } \\
\text { ATTENDEES }\end{array}$ & $\begin{array}{l}\text { NO. OF } \\
\text { SESSIONS }\end{array}$ \\
\hline & & & [62] & [63] \\
\hline A & Adelaide College of Divinity - Adelaide Theological Library & SA & 32 & 6 \\
\hline C & Alphacrucis College - James Wallace Memorial Library & NSW & & \\
\hline C & Australian Catholic University & AUST & 28522 & 973 \\
\hline A & Australian Lutheran College - Lohe Memorial Library & SA & 35 & 5 \\
\hline A & Benedictine Community New Norcia - New Norcia Library & WA & & \\
\hline A & Bible College of South Australia & SA & Oct-15 & 2 \\
\hline A & Booth College of Mission - The Salvation Army & NZ & 21 & \\
\hline A & Booth College - Booth College Library & NSW & 11 & 1 \\
\hline A & Camden Theological Library - Uniting Church in Australia & NSW & as required & \\
\hline C & Campion College - Campion College Library & NSW & & \\
\hline A & Carey Baptist College - Ayson Clifford Library & NZ & 145 & 16 \\
\hline B & $\begin{array}{l}\text { Catholic Education Office - Catholic Resource \& Information } \\
\text { Service }\end{array}$ & SA & 275 & 19 \\
\hline & Catholic Education Office of WA - Catholic Library of WA & WA & & \\
\hline A & Catholic Institute of Sydney - Veech Library & NSW & & \\
\hline A & Catholic Theological College - Mannix Library & $\mathrm{VIC}$ & & \\
\hline A & Christ College - Christ College Library & NSW & & \\
\hline $\mathrm{C}$ & Christian Heritage College - Christian Heritage College & QLD & 180 & 7 \\
\hline A & Divine World Missionaries - Patrick Murphy Memorial & $\mathrm{VIC}$ & as required & \\
\hline C & $\begin{array}{l}\text { Excelsia College - Gordon Moyes Library (formerly Wesley } \\
\text { Institute) }\end{array}$ & NSW & 200 & \\
\hline A & Good Shepherd College - Colin Library & NZ & 12 & 2 \\
\hline A & Harvest Bible College - QLD Library & QLD & & \\
\hline A & Harvest Bible College - VIC Library & VIC & & \\
\hline A & HarvestWest Bible College - Jenni Roche Memorial Library & WA & 30 & 2 \\
\hline A & $\begin{array}{l}\text { John Kinder Theological Library - Anglican Church in Aotearoa } \\
\text { New Zealand and Polynesia }\end{array}$ & NZ & 25 & 1 \\
\hline C & Lifeway College - David Yaxley Memorial Library & $\mathrm{NZ}$ & 160 & 30 \\
\hline A & $\begin{array}{l}\text { Malyon College (QLD Baptost College of Ministries) - } \\
\text { Malyon College Library }\end{array}$ & QLD & 45 & 3 \\
\hline A & Mary Andrews College & NSW & & \\
\hline A & Morling College - Gilbert Wright Library & NSW & & \\
\hline A & Nazarene Theological College - John D. Fulton Library & QLD & 20 & 10 \\
\hline A & Presbyterian Research Centre - Hewitson Library & $\mathrm{NZ}$ & & \\
\hline & Queensland Theological College - Gibson-Radclife Library & QLD & 59 & \\
\hline A & Ridley College - Leon Morris Library & VIC & 90 & 17 \\
\hline A & St Athanasius Coptic Orthodox Theological College - & $\mathrm{VIC}$ & & \\
\hline B & St Benedict's Monastery - St Benedicts Monastery Library & NSW & & \\
\hline A & St Francis Theological College - Roscoe Library & QLD & & \\
\hline & $\begin{array}{l}\text { St Mark's Anglican National Memorial Library - Charles } \\
\text { Sturt University }\end{array}$ & ACT & 230 & 15 \\
\hline & St Paschal Library - Franciscan Order of Friars Minor & VIC & 50 & 4 \\
\hline A & Sunshine Coast Theological College & QLD & & \\
\hline A & Sydney Missionary \& Bible College - Kerr Library & NSW & 279 & 181 \\
\hline C & Tabor College Tasmania - Tabor Library & TAS & & \\
\hline C & Tabor College Victoria & VIC & & \\
\hline & Trinity College - Leeper \& Mollison Libraries & VIC & & \\
\hline A & Triniy College - Trinity Theological Library & QLD & & \\
\hline A & Trinity Theological College & WA & 39 & 2 \\
\hline A & Vianney College - St Ann's Library & NSW & 8 & 3 \\
\hline A & Vose Seminary - Vose Library & WA & 150 & 9 \\
\hline A & Youthworks College - Youthworks College Library & NSW & & \\
\hline & & & 72896 & 1308 \\
\hline & RAGE & & 3037.333333 & 62.28571429 \\
\hline
\end{tabular}




\section{TABLE 92014 LIBRARY FACILITIES}

\begin{tabular}{|c|c|c|c|c|c|c|c|}
\hline \multirow[t]{2}{*}{$\begin{array}{l}0 \\
0 \\
\\
\end{array}$} & \multirow[t]{2}{*}{ INSTITUTION } & \multirow[t]{2}{*}{$\begin{array}{c}\text { STATE/N } \\
Z\end{array}$} & \multirow[t]{2}{*}{$\begin{array}{c}\text { TOTAL SEATING } \\
\text { CAPACITY }\end{array}$} & \multirow{2}{*}{ 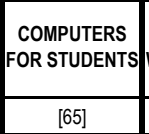 } & \multirow{2}{*}{$\begin{array}{c}\text { COMPUTERS } \\
\text { WITH INTERNET } \\
{[66]} \\
\end{array}$} & \multirow{2}{*}{$\begin{array}{c}\text { WIRELESS } \\
\text { AVAILABLE? } \\
{[67]}\end{array}$} & \multirow{2}{*}{$\begin{array}{c}\text { COMPUTER } \\
\text { DOCKING } \\
\text { STATIONS } \\
{[68]}\end{array}$} \\
\hline & & & & & & & \\
\hline A & Adelaide College of Divinity - Adelaide Theological Library & SA & 40 & 8 & 12 & Yes & \\
\hline $\mathrm{C}$ & Alphacrucis College - James Wallace Memorial Library & NSW & 57 & 10 & 13 & Yes & \\
\hline C & Australian Catholic University & AUST & 1900 & 620 & 737 & Yes & 113 \\
\hline A & Australian Lutheran College - Lohe Memorial Library & SA & 65 & 6 & 13 & Yes & 1 \\
\hline A & Benedictine Community New Norcia - New Norcia Library & WA & 50 & & & & \\
\hline A & Bible College of South Australia & SA & 20 & 5 & 10 & Yes & \\
\hline A & Booth College of Mission - The Salvation Army & NZ & 37 & 1 & 4 & $\mathrm{y}$ & 22 \\
\hline A & Booth College - Booth College Library & NSW & 27 & 7 & 7 & Yes & 10 \\
\hline A & Camden Theological Library - Uniting Church in Australia & NSW & 30 & 11 & 16 & Yes & 20 \\
\hline C & Campion College - Campion College Library & NSW & 36 & 17 & 19 & $\mathrm{y}$ & 2 \\
\hline A & Carey Baptist College - Ayson Clifford Library & NZ & 113 & 16 & 20 & Yes & 40 \\
\hline \multirow[t]{2}{*}{ B } & $\begin{array}{l}\text { Catholic Education Office - Catholic Resource \& Information } \\
\text { Service }\end{array}$ & SA & 20 & 3 & 5 & Yes & \\
\hline & Catholic Education Office of WA - Catholic Library of WA & WA & 28 & 2 & 5 & Yes & \\
\hline A & Catholic Institute of Sydney - Veech Library & NSW & 107 & 6 & 14 & Yes & \\
\hline A & Catholic Theological College - Mannix Library & VIC & 70 & 10 & 15 & $\mathrm{y}$ & 2 \\
\hline A & Christ College - Christ College Library & NSW & 93 & 4 & 6 & $\mathrm{y}$ & \\
\hline C & Christian Heritage College - Christian Heritage College & QLD & 115 & 27 & 33 & Yes & \\
\hline A & Divine World Missionaries - Patrick Murphy Memorial & VIC & 5 & & 2 & Yes & 2 \\
\hline C & $\begin{array}{l}\text { Excelsia College - Gordon Moyes Library (formerly Wesley } \\
\text { Institute) }\end{array}$ & NSW & 40 & 17 & 17 & Yes & 10 \\
\hline A & Good Sheperd College - Colin Library & NZ & 14 & 4 & 4 & Yes & 6 \\
\hline A & Harvest Bible College - QLD Library & QLD & 5 & 2 & 3 & No & \\
\hline A & Harvest Bible College - VIC Library & VIC & 9 & 8 & 9 & Yes & \\
\hline A & HarvestWest Bible College - Jenni Roche Memorial Library & WA & 10 & 10 & 10 & Yes & 10 \\
\hline A & $\begin{array}{l}\text { John Kinder Theological Library - Anglican Church in Aotearoa } \\
\text { New Zealand and Polynesia }\end{array}$ & NZ & 51 & 5 & 17 & Yes & \\
\hline C & Lifeway College - David Yaxley Memorial Library & NZ & 24 & 7 & 8 & Yes & 12 \\
\hline A & $\begin{array}{l}\text { Malyon College (QLD Baptost College of Ministries) - } \\
\text { Malyon College Library }\end{array}$ & QLD & 57 & 7 & 9 & Yes & 18 \\
\hline A & Mary Andrews College & NSW & 14 & 2 & 2 & No & \\
\hline A & Morling College - Gilbert Wright Library & NSW & 62 & 10 & 12 & Yes & \\
\hline A & Nazarene Theological College - John D. Fulton Library & QLD & 27 & 5 & 10 & Yes & 6 \\
\hline \multirow[t]{2}{*}{$\mathrm{A}$} & Presbyterian Research Centre - Hewitson Library & NZ & 58 & 4 & 4 & Yes & 2 \\
\hline & Queensland Theological College - Gibson-Radclife Library & QLD & 33 & 4 & 12 & Yes & \\
\hline A & Ridley College - Leon Morris Library & VIC & 80 & 10 & 14 & Yes & 26 \\
\hline A & St Athanasius Coptic Orthodox Theological College - & VIC & 8 & 3 & 4 & Yes & \\
\hline B & St Benedict's Monastery - St Benedicts Monastery Library & NSW & 20 & & 1 & No & 1 \\
\hline \multirow[t]{3}{*}{$\mathrm{A}$} & St Francis Theological College - Roscoe Library & QLD & 23 & 6 & 9 & $\mathrm{Y}$ & 2 \\
\hline & $\begin{array}{l}\text { St Mark's Anglican National Memorial Library - Charles } \\
\text { Sturt University }\end{array}$ & ACT & 15 & 5 & 11 & Yes & \\
\hline & St Paschal Library - Franciscan Order of Friars Minor & VIC & 44 & 3 & 8 & y & 6 \\
\hline A & Sunshine Coast Theological College & QLD & 4 & 1 & 2 & Yes & \\
\hline A & Sydney Missionary \& Bible College - Kerr Library & NSW & 90 & 7 & 12 & Yes & 55 \\
\hline $\mathrm{C}$ & Tabor College Tasmania - Tabor Library & TAS & 10-Dec & 2 & 4 & Yes & 6 \\
\hline \multirow[t]{2}{*}{$\mathrm{C}$} & Tabor College Victoria & VIC & 30 & 7 & 10 & Yes & 12 \\
\hline & Trinity College - Leeper \& Mollison Libraries & VIC & 120 & 13 & 17 & $\mathrm{y}$ & 28 \\
\hline A & Triniy College - Trinity Theological Library & QLD & 19 & 2 & 6 & Yes & \\
\hline A & Trinity Theological College & WA & 52 & 4 & 9 & Yes & 16 \\
\hline A & Vianney College - St Ann's Library & NSW & 17 & 6 & 8 & Yes & 16 \\
\hline A & Vose Seminary - Vose Library & WA & 40 & 7 & 11 & $\mathrm{y}$ & \\
\hline $\mathrm{A}$ & Youthworks College - Youthworks College Library & NSW & & & & & \\
\hline \multicolumn{3}{|c|}{ TOTAL } & 46127 & 914 & 1174 & & 444 \\
\hline \multicolumn{3}{|c|}{ AVERAGE } & 1002.76087 & 21.25581395 & 26.08888889 & & 17.07692308 \\
\hline
\end{tabular}


TABLE 102014 STAFF SALARIES

\begin{tabular}{|c|c|c|c|}
\hline INSTITUTION & PROF. & CHURCH & OTHER \\
\hline A & 17 & 5 & 7 \\
\hline B & & & 2 \\
\hline C & 4 & & 3 \\
\hline No Reporting Category & 2 & 1 & 2 \\
\hline TOTAL & 21 & 5 & 14 \\
\hline
\end{tabular}

\section{Staff Salaries}

2014

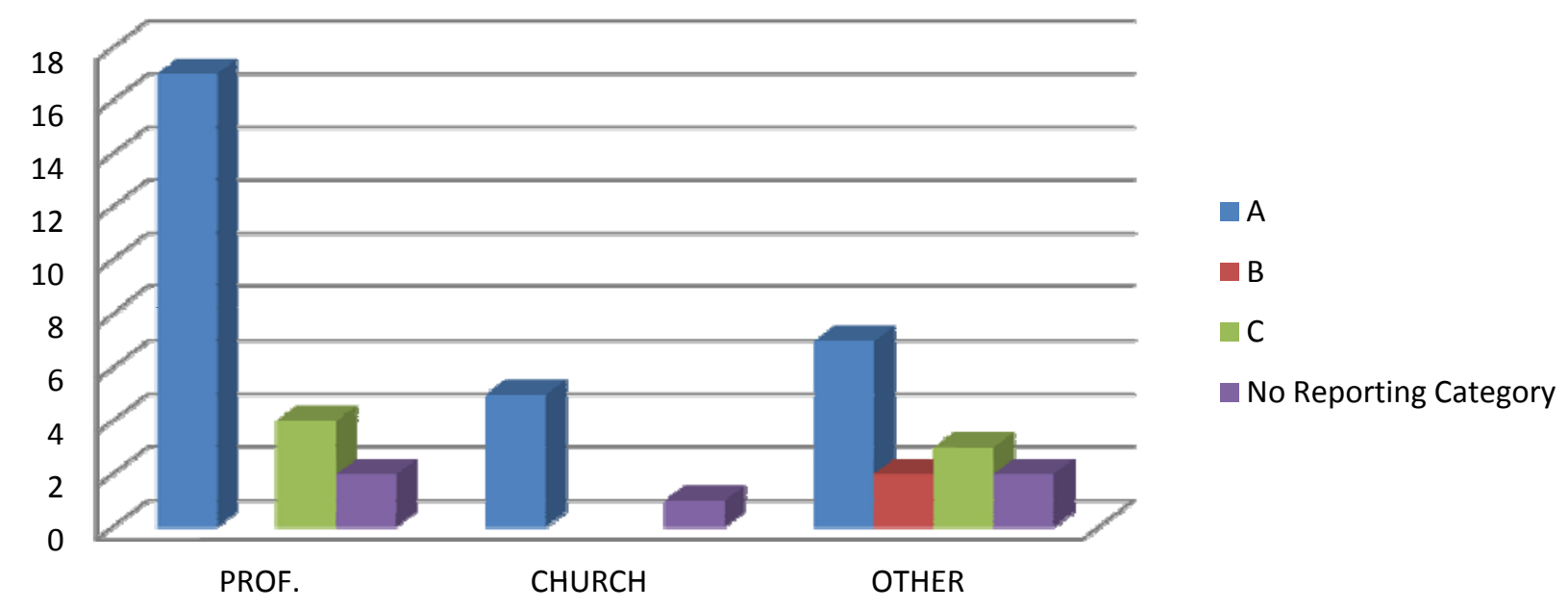


TABLE 112014 GENERAL

\begin{tabular}{|c|c|c|c|c|c|c|c|c|c|c|}
\hline \multirow[t]{2}{*}{$\begin{array}{ll}0 \\
\dot{m} \\
\&\end{array}$} & \multirow[t]{2}{*}{ INSTITUTION } & \multirow[t]{2}{*}{$\begin{array}{c}\text { STATE/N } \\
z\end{array}$} & \multirow[t]{2}{*}{ LIBRARY SYSTEM USED } & $\begin{array}{l}\text { SELF } \\
\text { CIRC }\end{array}$ & $A C Q$. & CIRC. & SERIALS & CAT. & $\begin{array}{l}\text { USED } \\
\text { OPAC }\end{array}$ & OTHER \\
\hline & & & & \multicolumn{7}{|c|}{ [71] } \\
\hline A & Adelaide College of Divinity - Adelaide Theological Library & SA & ALMA & & & $\mathrm{Y}$ & & $\mathrm{Y}$ & $\mathrm{Y}$ & \\
\hline C & Alphacrucis College - James Wallace Memorial Library & NSW & Liberty & $Y$ & $\mathrm{Y}$ & $\mathrm{Y}$ & $\mathrm{Y}$ & $\mathrm{Y}$ & $\mathrm{Y}$ & \\
\hline C & Australian Catholic University & AUST & Aleph 500 & $\bar{Y}$ & $\mathrm{Y}$ & $\bar{Y}$ & $\mathrm{Y}$ & $\bar{Y}$ & $\bar{Y}$ & \\
\hline A & Australian Lutheran College - Lohe Memorial Library & SA & Symphony & & & $\mathrm{Y}$ & & $\mathrm{Y}$ & $\mathrm{Y}$ & \\
\hline A & Benedictine Community New Norcia - New Norcia Library & WA & InMagic DB & & & & $\mathrm{Y}$ & $\mathrm{Y}$ & $\bar{Y}$ & \\
\hline A & Bible College of South Australia & SA & Liberty & Y & & $\mathrm{Y}$ & & $\mathrm{Y}$ & $\mathrm{Y}$ & \\
\hline A & Booth College of Mission - The Salvation Army & NZ &.$e L m$ & $\mathrm{Y}$ & $\mathrm{Y}$ & $\bar{Y}$ & & $\bar{Y}$ & $\mathrm{Y}$ & \\
\hline A & Booth College - Booth College Library & NSW & Liberty 5 & $\mathrm{Y}$ & $\bar{Y}$ & $\bar{Y}$ & & $\bar{Y}$ & $\mathrm{Y}$ & Federated search \\
\hline $\mathrm{A}$ & Camden Theological Library - Uniting Church in Australia & NSW & Liberty 5 & & $\mathrm{Y}$ & $\bar{Y}$ & $\mathrm{Y}$ & $\bar{Y}$ & $\bar{Y}$ & \\
\hline C & Campion College - Campion College Library & NSW & Aleph & & $\mathrm{Y}$ & $\mathrm{Y}$ & $\mathrm{Y}$ & $\mathrm{Y}$ & $\bar{Y}$ & \\
\hline $\mathrm{A}$ & Carey Baptist College - Ayson Clifford Library & $\mathrm{NZ}$ & Liberty 5 & & $\mathrm{Y}$ & $\mathrm{Y}$ & $\mathrm{Y}$ & $\mathrm{Y}$ & $\mathrm{Y}$ & \\
\hline $\mathrm{B}$ & $\begin{array}{l}\text { Catholic Education Office - Catholic Resource \& Information } \\
\text { Service }\end{array}$ & SA & Concord Infiniti & & & $\mathrm{Y}$ & & $\mathrm{Y}$ & $\mathrm{Y}$ & \\
\hline & Catholic Education Office of WA - Catholic Library of WA & WA & & & $\mathrm{Y}$ & & $\mathrm{Y}$ & $\bar{Y}$ & & \\
\hline A & Catholic Institute of Sydney - Veech Library & NSW & Aleph & $\mathrm{Y}$ & $\mathrm{Y}$ & $\mathrm{Y}$ & $\mathrm{Y}$ & $\mathrm{Y}$ & $\mathrm{Y}$ & \\
\hline A & Catholic Theological College - Mannix Library & VIC & Horizon & & & $\mathrm{Y}$ & & $\mathrm{Y}$ & $\mathrm{Y}$ & Discovery Layer \\
\hline A & Christ College - Christ College Library & NSW & Access-It & $\mathrm{Y}$ & & $\mathrm{Y}$ & & $\mathrm{Y}$ & $\mathrm{Y}$ & \\
\hline C & Christian Heritage College - Christian Heritage College & QLD & \multicolumn{2}{|l|}{ ELibris-Aleph / PRIMO } & $\mathrm{Y}$ & $\mathrm{Y}$ & & $\mathrm{Y}$ & $\mathrm{Y}$ & \\
\hline A & Divine World Missionaries - Patrick Murphy Memorial & VIC & & & & & & & & \\
\hline C & $\begin{array}{l}\text { Excelsia College - Gordon Moyes Library (formerly Wesley } \\
\text { Institute) }\end{array}$ & NSW & Liberty & & Y & $\mathrm{Y}$ & $\mathrm{Y}$ & $\mathrm{Y}$ & $\mathrm{Y}$ & \\
\hline A & Good Shepherd College - Colin Library & NZ & $\mathrm{eLm}$ & & $\mathrm{Y}$ & $\mathrm{Y}$ & & $\mathrm{Y}$ & $\mathrm{Y}$ & \\
\hline A & Harvest Bible College - QLD Library & QLD & \multirow{2}{*}{ Koha } & \multirow{2}{*}{ Y } & \multirow{2}{*}{$\mathrm{Y}$} & \multirow{2}{*}{ Y } & \multirow{2}{*}{$\mathrm{Y}$} & \multirow{2}{*}{$\mathrm{Y}$} & \multirow{2}{*}{$\mathrm{Y}$} & \\
\hline A & Harvest Bible College - VIC Library & VIC & & & & & & & & \\
\hline A & HarvestWest Bible College - Jenni Roche Memorial Library & WA & Bookmark & Y & & $\mathrm{Y}$ & & $\mathrm{Y}$ & $\mathrm{Y}$ & \\
\hline $\mathrm{A}$ & $\begin{array}{l}\text { John Kinder Theological Library - Anglican Church in Aotearoa } \\
\text { New Zealand and Polynesia }\end{array}$ & NZ & elm & & $\mathrm{Y}$ & $\mathrm{Y}$ & & $\mathrm{Y}$ & $\mathrm{Y}$ & \\
\hline C & Lifeway College - David Yaxley Memorial Library & $\mathrm{NZ}$ & Access-It & & $\mathrm{Y}$ & $\mathrm{Y}$ & $\mathrm{Y}$ & $\mathrm{Y}$ & $\mathrm{Y}$ & \\
\hline $\mathrm{A}$ & $\begin{array}{l}\text { Malyon College (QLD Baptost College of Ministries) - } \\
\text { Malyon College Library }\end{array}$ & QLD & Liberty 5 & $\mathrm{y}$ & $\mathrm{y}$ & $\mathrm{y}$ & $\mathrm{y}$ & $y$ & $\mathrm{y}$ & |ILLs \\
\hline $\mathrm{A}$ & Mary Andrews College & NSW & Filemaker Pro & & & & & & & \\
\hline A & Morling College - Gilbert Wright Library & NSW & Liberty 5 & $\mathrm{Y}$ & $\mathrm{Y}$ & $\mathrm{Y}$ & $\mathrm{Y}$ & $\mathrm{Y}$ & $\mathrm{Y}$ & \\
\hline A & Nazarene Theological College - John D. Fulton Library & QLD & Destiny & & & & & & $\mathrm{Y}$ & \\
\hline \multirow[t]{2}{*}{ A } & Presbyterian Research Centre - Hewitson Library & NZ & Koha & & & $\mathrm{Y}$ & $\mathrm{Y}$ & $\mathrm{Y}$ & $\mathrm{Y}$ & \\
\hline & Queensland Theological College - Gibson-Radclife Library & QLD & Liberty 5 & Y & & $\mathrm{Y}$ & & $\mathrm{Y}$ & $\mathrm{Y}$ & \\
\hline $\mathrm{A}$ & Ridley College - Leon Morris Library & VIC & Horizon & & & $\mathrm{Y}$ & & $\mathrm{Y}$ & $\mathrm{Y}$ & \\
\hline $\mathrm{A}$ & St Athanasius Coptic Orthodox Theological College - & VIC & Koha & & & $\mathrm{Y}$ & & $\mathrm{Y}$ & $\mathrm{Y}$ & Reports \\
\hline $\mathrm{B}$ & St Benedict's Monastery - St Benedicts Monastery Library & NSW & LibraryWorld & & & $\mathrm{Y}$ & & & $\mathrm{Y}$ & Reports \\
\hline \multirow[t]{3}{*}{ A } & St Francis Theological College - Roscoe Library & QLD & Liberty 5 & $\mathrm{Y}$ & $\mathrm{Y}$ & $\mathrm{Y}$ & $\mathrm{Y}$ & $\mathrm{Y}$ & $\mathrm{Y}$ & ILLs \\
\hline & $\begin{array}{l}\text { St Mark's Anglican National Memorial Library - Charles } \\
\text { Sturt University }\end{array}$ & ACT & & Y & $\mathrm{Y}$ & Y & $\mathrm{Y}$ & Y & Y & \\
\hline & St Paschal Library - Franciscan Order of Friars Minor & VIC & Liberty 5 & & $\mathrm{Y}$ & $\mathrm{Y}$ & $\mathrm{Y}$ & $\mathrm{Y}$ & $\mathrm{Y}$ & Management \\
\hline A & Sunshine Coast Theological College & QLD & Library Master & & & $\mathrm{Y}$ & & $\mathrm{Y}$ & & \\
\hline A & Sydney Missionary \& Bible College - Kerr Library & NSW & Liberty 5 & $\mathrm{Y}$ & $\mathrm{Y}$ & $\mathrm{Y}$ & $\mathrm{Y}$ & $\mathrm{Y}$ & $\mathrm{Y}$ & \\
\hline $\mathrm{C}$ & Tabor College Tasmania - Tabor Library & TAS & Bookmark 10 & $\mathrm{Y}$ & & $\mathrm{Y}$ & & $\mathrm{Y}$ & $\mathrm{Y}$ & \\
\hline \multirow{2}{*}{ C } & Tabor College Victoria & $\mathrm{VIC}$ & Destiny & $\mathrm{Y}$ & & $\mathrm{Y}$ & & $\mathrm{Y}$ & $\mathrm{Y}$ & \\
\hline & Trinity College - Leeper \& Mollison Libraries & VIC & Symphony Workflows & $\mathrm{Y}$ & & $\mathrm{Y}$ & $\mathrm{Y}$ & $\mathrm{Y}$ & $\mathrm{Y}$ & Reports \& Digital Asset Management \\
\hline A & Triniy College - Trinity Theological Library & QLD & \begin{tabular}{|l|l} 
Liberty 5 & \\
\end{tabular} & $\mathrm{Y}$ & $\mathrm{Y}$ & $\mathrm{Y}$ & $\mathrm{Y}$ & $\mathrm{Y}$ & $\mathrm{Y}$ & ILLs, Z Cataloguing; Syndetics Sol'ns \\
\hline A & Trinity Theological College & WA & OCLC Worldshare & $\mathrm{Y}$ & $\mathrm{Y}$ & $\mathrm{Y}$ & & $\mathrm{Y}$ & $\mathrm{Y}$ & Discovery Layer \\
\hline $\mathrm{A}$ & Vianney College - St Ann's Library & NSW & Destiny & $\mathrm{Y}$ & & $\mathrm{Y}$ & & $\mathrm{Y}$ & $\mathrm{Y}$ & \\
\hline $\mathrm{A}$ & Vose Seminary - Vose Library & WA & Liberty & $Y$ & & $\mathrm{Y}$ & $\mathrm{Y}$ & $\mathrm{Y}$ & $\mathrm{Y}$ & \\
\hline$A$ & Youthworks College - Youthworks College Library & NSW & & & & & & & & \\
\hline
\end{tabular}




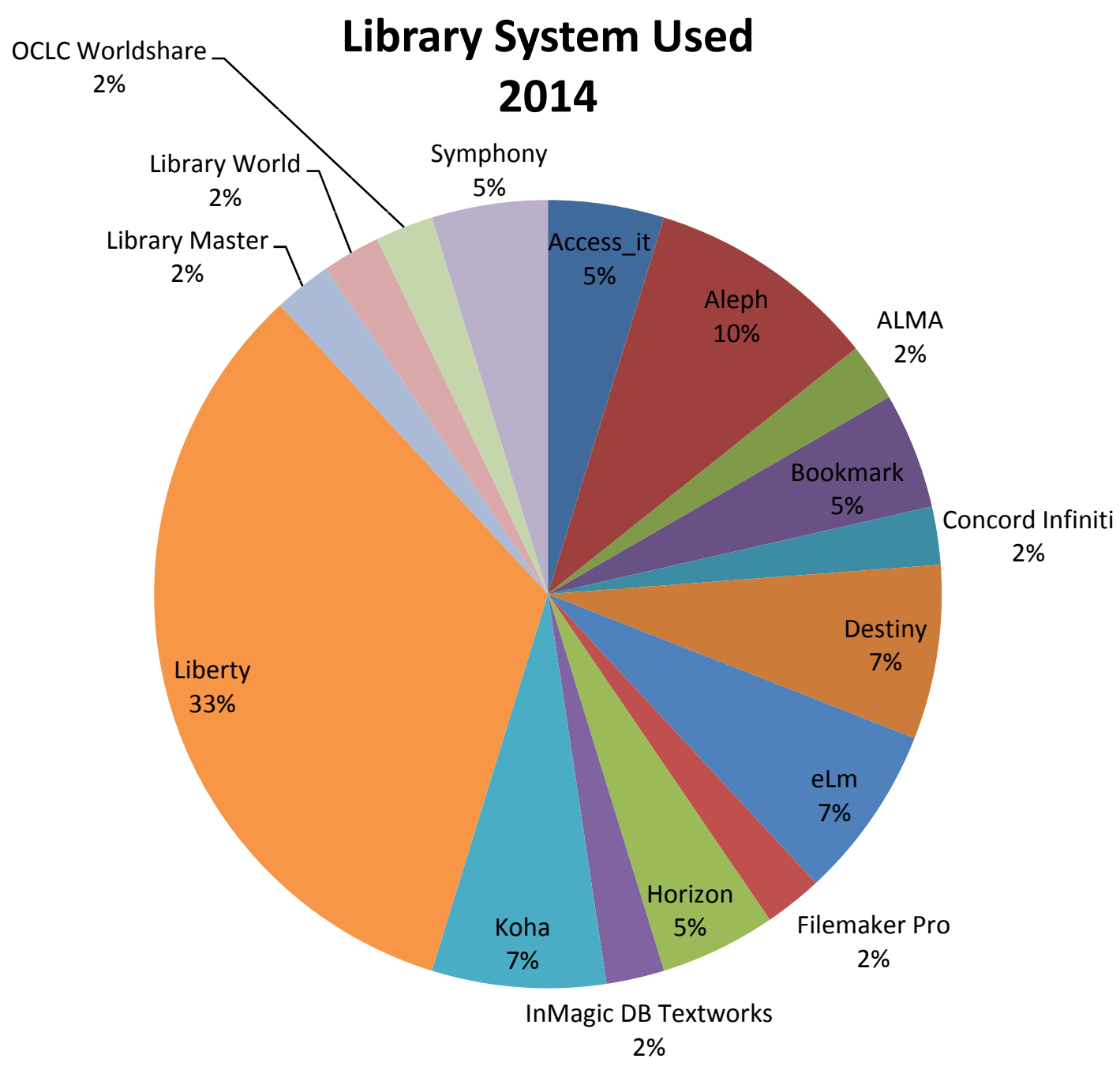




\section{Contributors}

Hayley Eyers completed her Diploma in Library and Information Studies at TAFESA in 2007. She juggles two part-time library jobs. Hayley is a library technician at the Adelaide Theological Library. Her other job is as an Information Services Officer at the Tea Tree Gully TAFESA Library. Tea Tree Gully TAFESA Library is part of the TAFESA Adelaide North Libraries, for which Hayley has worked for more than five years. Hayley's interests outside the library world are hummingbirds, beading, crafts, kickboxing, and much more.

Kerrie Stevens has always wanted to be a librarian and is currently living this dream at Harvest Bible College in Melbourne. Previous employment opportunities have included the CSIRO (in the food science area), Stockdale Prospecting (a diamond mining company) and La Trobe University - all within their libraries. Since joining ANZTLA in 2003, Kerrie has held various positions, and loves the co-operative spirit within the association. Kerrie is addicted to cross-stitch; loves her Golden Retriever, Clarence; and has a very, very sweet tooth. 